\title{
Analysing experiences and issues in self-built shelters in Bangladesh using transdisciplinary approach
}

\author{
Tonja Klansek ${ }^{1}$. David A. Coley ${ }^{2}$ - Natalia Paszkiewicz ${ }^{3}$ - Dima Albadra² ${ }^{2}$. \\ Federico Rota $^{4} \cdot$ Richard J. Ball ${ }^{2}$
}

Received: 8 April 2020 / Accepted: 25 September 2020 / Published online: 8 October 2020

(C) The Author(s) 2020

\begin{abstract}
There are currently 70.8 million forcibly displaced people, globally. Bangladesh hosts the largest refugee camp in the world. Much effort has gone into the research, design and delivery of mass-produced shelters. Yet most refugees live in self-built shelters using simple shelter materials. This paper aims to demonstrate the benefits of using a transdisciplinary approach for holistic data collection in such shelters. A total of 1594 households were surveyed in refugee camps in Bangladesh using diverse methods-e.g. surveys, semi-structured interviews, physical measurements. It was only because of the use of various methods that the reasons behind identified issues were discovered or quantified. For example, household surveys uncovered the issue of poor ventilation, but only the semi-structured interviews exposed the reasons behind it, while physical measurements assessed the implications of this-annual particulate exposure 13 times the recommended limit. Furthermore, several methods pointed to issues with materials, but only the focus groups discussions exposed the need for gender-sensitive technical training tailored for women on the correct use of the materials. This study demonstrates that a diverse team (humanitarian staff, building physicists, and anthropologists) using several approaches to data-gathering and working in a transdisciplinary manner has much to offer the sector, and by including quantitative physical measurements allows costed improvement plans to be developed, targets to be set and general, rather than case specific, knowledge to be generated. The findings of this study have resulted in new shelter interventions by the aid sector that were rolled out in over 70,000 shelters.
\end{abstract}

Keywords Temporary shelters $\cdot$ Refugee camps $\cdot$ Bangladesh $\cdot$ Transdisciplinary approach

Dima Albadra

d.albadra@bath.ac.uk

1 Shelter/NFI Sector Cox's Bazar, International Organisation for Migration (IOM), Geneva, Switzerland

2 Department of Architecture and Civil Engineering, University of Bath, Bath, UK

3 Department of Social and Policy Sciences, University of Bath, Bath, UK

4 Catholic Relief Services (CRS), Baltimore, USA 


$\begin{array}{ll}\text { Abbreviations } \\ \text { USK } & \text { Upgrade shelter kits } \\ \text { ESK } & \text { Emergency shelter kits } \\ \text { TDK } & \text { Tie-down kits } \\ \text { FGD } & \text { Focus group discussions } \\ \text { HLA } & \text { Household level assessment } \\ \text { SSI } & \text { Semi-structured interviews } \\ \text { PM } & \text { Particulate matter } \\ \text { VOCs } & \text { Volatile organic matter }\end{array}$

\section{Introduction}

As of 2019 there were 70.8 million forcibly displaced people worldwide; an increase of 2.3 million people over the previous year (UNHCR 2020a). Pomponi et al. 2019 points out that most academic papers concerned with refugee camps and temporary shelters focus on either technical aspects (for instance, Chowdhury et al. 2020) or social aspects (Hart et al. 2018). Furthermore, most technical studies are focused on design and delivery of mass-produced shelters, e.g. Wang et al. (2017), D'Orazio et al. (2020), Atmaca and Atmaca (2016). Studies that contain temperature and air quality assessments in real settings and inhabited shelters are far less common, particularly of self-built accommodation. Studies such as, Fosas et al. (2018) and Albadra et al. (2017, 2018) in Zaartari and Azraq refugee camps in Jordan, revealed significant overheating and occupant dissatisfaction. These shelters were costly (by aid agency standards) mass-produced solutions rather than self-built, so might be expected to provide better environmental conditions than simple, low-cost, self-built shelters. That conditions were poor in this relatively expensive, engineered accommodation, suggested the urgent need to assess the self-built sector approach. Alshawawreh et al. (2017), and Albadra et al. (2017) also found that significant adaptations had been made to the Zaatari shelters by the occupants. This suggests that, in theory, selfdetermined and hence self-built accommodation has the potential to enhance living conditions and occupant satisfaction. This fits well with the general desire in the humanitarian community to move more toward self-built shelter solutions with shelter kits and/or cash/ vouchers for materials schemes (Maynard et al. 2016).

This paper reports on an investigation in Cox's Bazar, Bangladesh conducted by the aid sector and academics aimed at: (1) producing a generalised transdisciplinary methodology for assessing and analysing issues with self-built shelter; (2) demonstrating it at a large scale (1594 households); (3) analysing any issues found with the bamboo-framed, tarpaulin-covered shelters.

\subsection{Setting}

Bangladesh hosts almost one million refugees, with more than 723,000 Rohingya refugees crossing the borders from Myanmar since August 2017 (UNHCR 2020b). The majority have settled in makeshift settlements and spontaneous camps near Cox's Bazar (Fig. 1), in self-built shelters with bamboo and tarpaulin bought or supplied upon arrival.

In 2018 the shelter aid sector carried out the distribution of Upgrade Shelter Kits (USK) and Tie Down Kits (TDK) (see "Appendix 1") used to complement the Emergency Shelter Kits (ESK) that had been the foundation of the camp. The ESK was meant to provide 


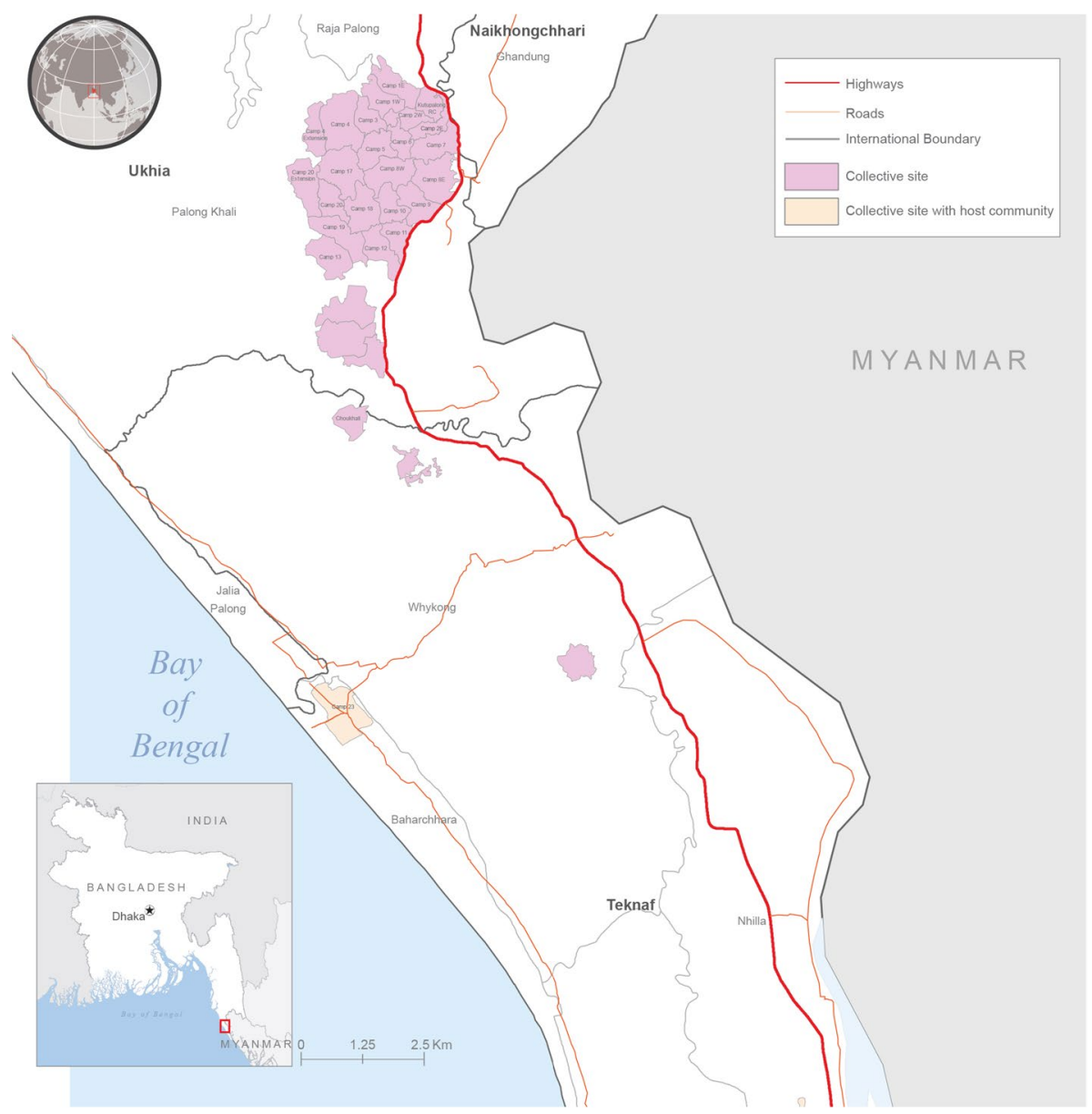

Fig. 1 Map adapted from ISCG (2020), illustrating the location of refugee camps 'collective sites' in Bangladesh

immediate life-saving assistance during the acute phase of the emergency and consisted of two tarpaulins, one bundle of rope, four pieces of big bamboo and sixty pieces of small bamboo. Families used these materials to erect simple shelters to protect them from the elements. Subsequently, USK was distributed to support families improving the existing structures with additional shelter materials. The USK distribution was coupled with the issuing of technical guidance for beneficiaries to build safer shelters and the provision of localized household level site improvements that will enhance living conditions and contribute to disaster risk reduction. The intention of the USK were to upgrade and strengthen shelters that were constructed with ESK materials provided in Phase 1 of the shelter response. By August 2018, more than 212,000 households had received a USK. The TDK kit was developed slightly later as an addition to the USK and is intended for protection of the shelters against the seasonal climate changes in the area, especially high winds. (The climate is classified as tropical with the average annual temperature is $25.6{ }^{\circ} \mathrm{C}$ and the average annual rainfall is $3770 \mathrm{~mm}$.) Fig. 2 shows a typical self-built shelter consisting of 

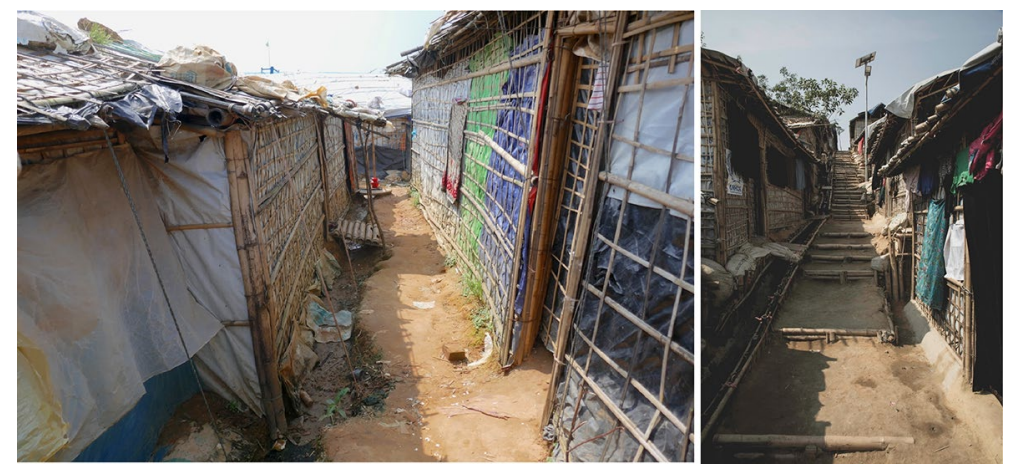

Fig. 2 The main construction and shelter spacing in Cox's Bazar

a tarpaulin-covered bamboo frame. This research was carried out after the distribution of these kits to provides evidence of the true use of such kits.

\section{Methodology}

Quantitative and qualitative methods were used to assess the performance of the shelters and gather the refugees' perspective, and to allow the transdisciplinary team to show its strengths. The study consisted of seven elements: (i) large-scale household level assessments (HLA), completed by general aid agency staff; (ii) semi-structured interviews (SSI) with 44 households, one family at a time, by an academic anthropologist; (iii) technical field visits to look at the structure of the shelters, by aid agency engineering staff; (iv) focus group discussions (FGD) with technical/field shelter staff, by general aid agency staff; (v) focus group discussions (FGD) with beneficiaries, by general aid agency staff; (vi) measurements of humidity, temperature and air quality in the shelters, by academic building physicists; (vii) Satellite imagery to determine the average shelter size, and whether Sphere Standard of a minimum $3.5 \mathrm{~m} 2$ of covered living space /person were satisfied.

The research conducted by the shelter aid sector (HLA, technical visits, and FGD) is discussed in detail in (IOM 2018).

The HLA was conducted to gather information on households' perspectives of their shelter. It was conducted in 1,594 households across 26 camps between 23rd of July and 7th of August 2018. The questionnaire was formulated in English (see "Appendix 2") and translated into Bangla then conducted in Chittagonian dialect. It consisted of the following sections: (i) Household information (8 questions and sub-questions when relevant); (ii) Current shelter and shelter materials (8 questions and sub-questions when relevant); (iii) Future shelter (4 questions and sub questions when relevant). In addition, during HLA participants were asked to identify the top three issues they face with their shelter. They could select between fifteen options as well as option "other' that could then be further described in a sub question.

The semi-structured interviews (SSI) were conducted by researchers from the University of Bath who visited the camps one year after the 2017 Rohingya exodus, and then returned to Cox's Bazar in the summer of 2019. Forty-four refugee households were interviewed in 12 camps, namely: Camp 8 East; Shamlapur; Kutupalong Registered; 
Camp 20 Extension; Camp 7; Camp 4 Extension; Camp 4; Unchiprang; Camp 16; Camp 2; Camp 3; Camp 27. Each interview lasted for about an hour and followed a questionnaire structure exploring respondents' satisfaction with various aspects of their shelters, such as structural safety, protection against elements, thermal comfort and others, alongside their priorities regarding shelter design. This was supported by observation of adaptations made by inhabitants and followed by more in-depth questions examining notions of privacy and security (see "Appendix 3"). This approach allowed occupants to discuss things in detail and to identify issues that might not emerge when using a structured survey method of only predefined questions, or a focus group. The approach used by the interviewer was an emic one, in this case asking detailed follow up questions. An emic approach which is traditionally used in ethnography through immersion in the field (Kahn 2014) seeks to capture the point of view of research participants and communicate the particularities of a given cultural context. An etic perspective on the other hand is applied by researchers who remain objective outsiders and focus on universalities when exploring a phenomenon (Beals et al. 2020).

The technical field visits by aid agencies assessed 34 shelters (two shelters randomly selected per camp). The data collected included the size of shelters, quality of construction and state of existing materials.

The focus group discussions with technical/field shelter staff focused on the perceptions of the staff that were implementing the shelter interventions. The FGD with technical staff was aimed at discussing USK implementation and suggested improvements for future interventions. In total, three FGDs were held. All the attendees were local staff members. Each FGD consisted of 18 questions, see "Appendix 4".

The focus group discussions with beneficiaries were aimed at providing more in depth understanding of the issues households face in regards to shelter and possible improvements. A total of 24 FGDs with beneficiaries were conducted 26th July and 6th of August 2018: female (6), male (10), extremely vulnerable females (5) and extremely vulnerable males (3) in groups ranging from 10 to 20 participants. In preparation of the FGDs, three pilot FGDs were conducted to ensure validity of questions asked. The FGD lasted approximately $1.5 \mathrm{~h}$ each and consisted of 19 questions. Questions were prepared in English (see "Appendix 5") and translated to Bangla. Questions covered: Shelter materials received; shelter materials purchased; training on shelter upgrades; current shelter condition; desired future shelter improvements/materials; community knowledge of alternative materials/ improvements (including mud walls) and main shelter concerns. Survey participants were then asked what their main concern was with regards to their current shelter. This metric was used in order to determine what improvements future shelter assistance may need to focus on as well as to better understand what households perceive as their main concern compared to technical/field staff.

Air temperature was monitored in nine shelters at $1.5 \mathrm{~m}$ above floor level for eleven days from 28th September 2018, using I-button sensors that were placed approximately in the centre of the shelter.

Air quality in the shelters was evaluated using a TSI DustTrak DRX Desktop Aerosol Monitor over a sampling period of 30 min to measure the level of Particulate Matter (PM). In addition, Volatile organic compounds (VOCs) were measured through active sampling for a period of $30 \mathrm{~min}$. Air from within the shelter interior was drawn through Tenax tubes using a calibrated sampling pump according to the Standard EN ISO 16,000-6:2011. This effectively trapped VOCs with boiling points between 60 and $280{ }^{\circ} \mathrm{C}$ on the Tenax adsorbent tubes which were later thermally desorbed and quantitatively analysed by gas chromatography. Major compounds were identified by mass spectrometry. 
The Open Street Map images were hand digitized through a desk-based process and did not include field verification. Several small structures that are non-shelters, latrines as an example, were included, larger structures such as schools and NGO offices were not included. Additionally, internal partitions of the shelters cannot be assessed and taken into account. Average covered space per person was calculated by dividing total covered area with total population of the camp. The method will overestimate the covered area and hence the size of the shelters and the floor area per occupant.

\section{Results}

The findings from the HLA, semi-structured interviews, technical field visits, focus groups and measurement were combined to give an overview of the situation in the camps, and the main issues found in self-built bamboo shelters. These issues ranged from structural and safety concerns, to comfort and air quality issues, to functional and sociocultural:

\subsection{Main concerns of occupants}

It is clear that the different assessment methods brought different issues to the fore. During the FGDs with households the issue of shelter size was raised as one of the main concerns, unlike FGDs with technical staff who did not raise this issue (Table 1). Unfortunately, the question of size was not included in the HLA. It was clear in the answers collected during the HLA that the main concerns were mostly related to the state of materials or the strength

Table 1 Main shelter concerns-comparison of findings from FGDs with Households and technical/field shelter staff (Shelter Survey July/August 2018)

\begin{tabular}{|c|c|}
\hline FGDs with technical/field shelter staff & FGDs with households \\
\hline- & Shelter is too small for large families \\
\hline- & Tarpaulin is old and damaged \\
\hline \multicolumn{2}{|l|}{$\begin{array}{l}\text { Lack of ventilation and smoky interior due to the use } \\
\text { of wood as a cooking fuel }\end{array}$} \\
\hline Heat in shelter during sunny days & Lack of ventilation and high temperature in shelters \\
\hline Cooking in shelter (fire hazard and smoke) & No cooking and bathing space \\
\hline Floor becomes damp & Shelter/floor becomes wet during the rain \\
\hline No fire safety measures in entire camp & $\begin{array}{l}\text { Space around shelter is very small, also congestion } \\
\text { of the camps }\end{array}$ \\
\hline \multicolumn{2}{|l|}{$\begin{array}{l}\text { Bamboo posts are inserted in the ground and will rot } \\
\text { within } 6 \text { months }\end{array}$} \\
\hline $\begin{array}{l}\text { Bamboo was not treated and was immature in several } \\
\text { cases }\end{array}$ & Bamboo is damaged and infested with borers \\
\hline Insufficient drainage & - \\
\hline \multicolumn{2}{|l|}{$\begin{array}{l}\text { Rafter and purlin ties are not strong due to insufficient } \\
\text { thickness of rope }\end{array}$} \\
\hline Tie down is not properly done or missing & Fear of landslide and cyclone \\
\hline Lack of locks (security) & $\begin{array}{l}\text { Additional partitions needed to add to privacy of } \\
\text { women and girls (some HHs do not have parti- } \\
\text { tions) }\end{array}$ \\
\hline
\end{tabular}




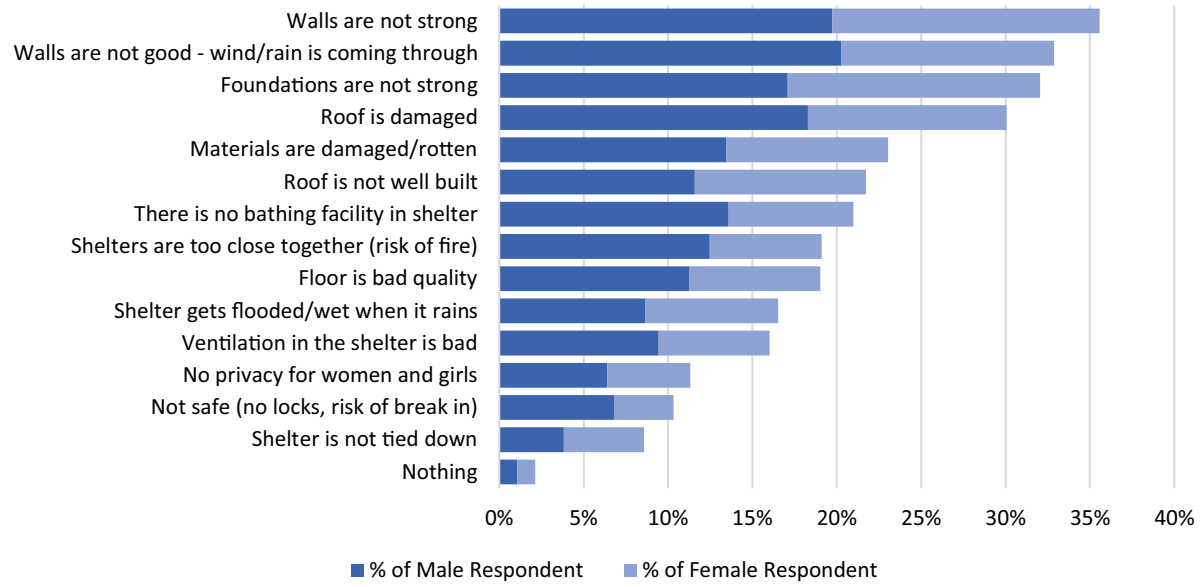

Fig. 3 Top three shelter concerns-Household Level Assessment

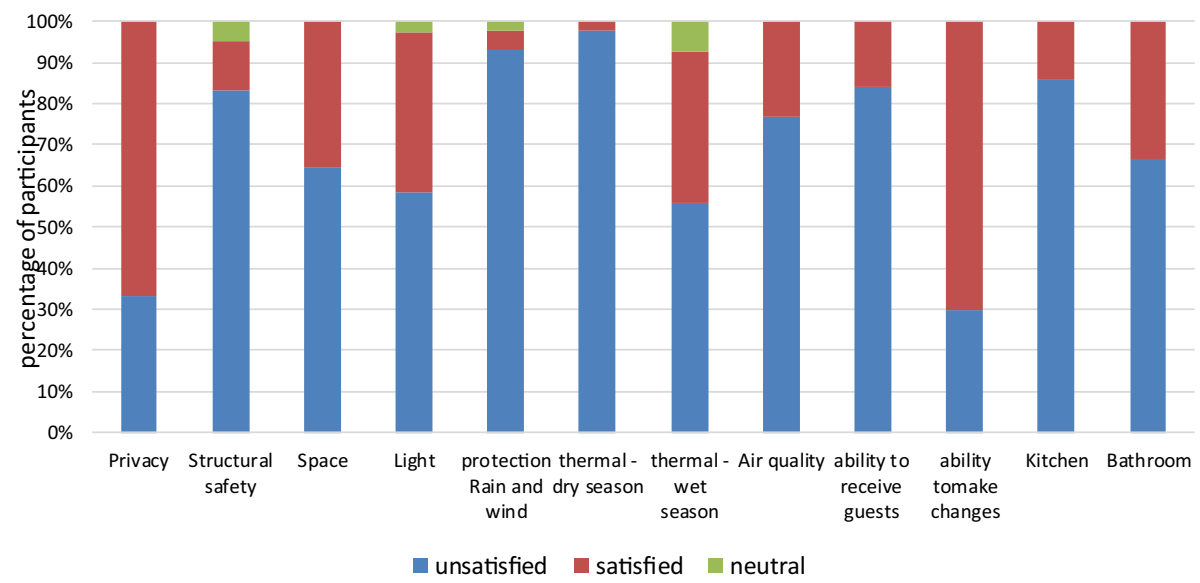

Fig. 4 Satisfaction levels with different shelter design aspects from SSI

of the shelter, although lack of bathing and cooking space, safety, and privacy arose as a main concern for some (Fig. 3). However, during SSI, thermal conditions were a top priority for the refugees (as indicated by 18 people out of 44 ), followed by appearance ( 7 participants) and space (5 participants). Privacy was the least important aspect of shelter design as indicated by nine participants, followed by security (six participants). It is worth noting here that refugees tend not to prioritise aspects already present in their accommodation; therefore, it possibly implies that privacy and security have been achieved in the case of those respondents.

Furthermore, during the SSIs, participants were asked about satisfaction with their shelters (Fig. 4). Participants were least satisfied with the thermal conditions during the dry season (i.e. overheating); followed by protection from rain and wind. Dissatisfaction levels were also high with regards to the structural safety of the shelters, cooking space and lack of ability to socialise and receive guests. 


\subsection{Occupancy density}

Based on technical field visits, on average, there were 5.5 people per household with an average shelter area of $20.8 \mathrm{~m} 2$, suggesting $3.8 \mathrm{~m} 2$ per person, while 12 shelters provide less than $3.5 \mathrm{~m} 2$ of covered area per person. Given that the minimum $3.5 \mathrm{~m} 2$ per person is a key target used by aid agencies - despite it being an arbitrary one as pointed out by Alshawawreh et al. (2020)_and that approximately one-third of shelters did not seem to be meeting the target, a larger sample size is suggested. Therefore, the average covered space was determined using Open Street Map satellite pictures. As indicated in the Methodology, this approach is likely to overestimate the floor area per person. The result varied greatly between camps from an average covered area of $1.33 \mathrm{~m} 2 /$ person to $7.41 \mathrm{~m} 2 /$ person (mean $\pm \mathrm{SD}=4.33 \pm 1.4 \mathrm{~m} 2 /$ person) (Fig. 5).

In conclusion, although covered space is $>3.5 \mathrm{~m} 2$ per person, as assessed by technical visits and satellite imagery, approximately a third of the residents are housed in smaller shelters than this. Furthermore, the proximity of shelters to each-others with no safe spacing results in an overall overcrowding in the camp, regardless of the covered space per person. External space was listed as important for 40 out of 44 respondents interviewed. Refugees reported having verandas in their houses in Myanmar, but this is something difficult to achieve in very congested refugee camps in Bangladesh. People wished to use private external space to have some rest away from the shelter's heat, to provide space for their children to play and to receive guests. It is clear from the satellite imagery analysis and images such as Fig. 2, that there is little space within the camp to increase the covered area per person or to provide any private external space.

\subsection{Shelter structure}

Information on the shelter structure was collected during the technical field visits and FGD with technical staff. The shelter structure was analysed to better understand how well shelters were constructed, culturally accepted practices, and possible future improvements.

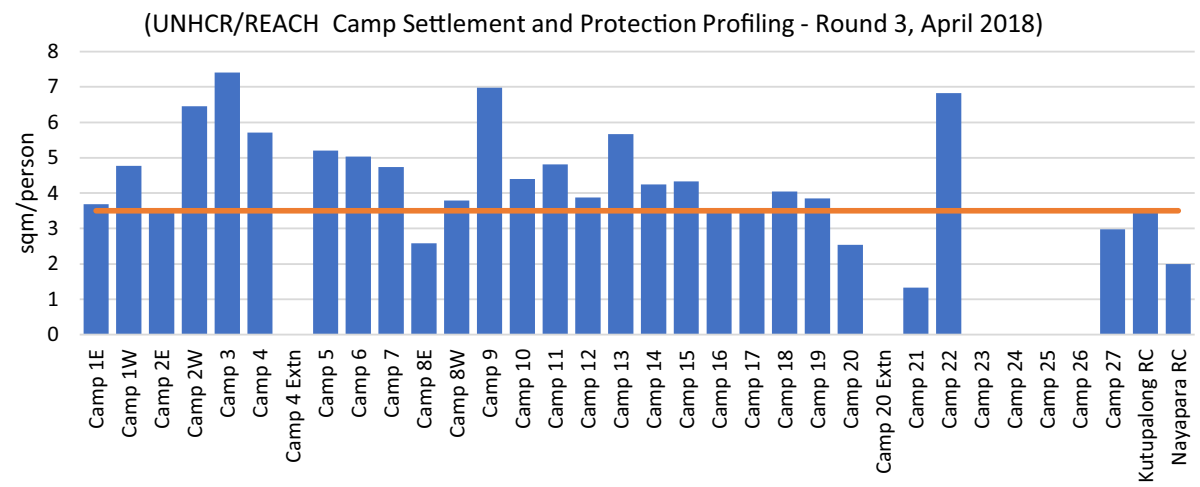

Fig. 5 Average covered area per person (UNHCR \& REACH 2018). The orange line shows the Sphere guidance (Sphere 2018) value of $3.5 \mathrm{~m}^{2}$ per person 


\subsubsection{Foundations}

The foundations (being underground) could not be directly observed, so the data was collected by asking the households who indicated the depth as a distance between the fingertips, elbow, or shoulder. The accuracy of these distances could not be verified. $53 \%$ of shelters did not have a cross anchor and in $41 \%$ of shelters the foundations were less than $30 \mathrm{~cm}$. It was noted during the FGDs with technical/field staff that one of the main shelter concerns is bamboo direct contact with the ground. Ensuring quality foundations that separates contact between bamboo and ground level should be flagged as a priority for future interventions, in addition to the foundations' depth.

\subsubsection{Plinth}

An earthen plinth (raised floor) was observed in all but 7 shelters. The height varied between 10 and $50 \mathrm{~cm}$ (mean $22 \mathrm{~cm}$ ). In $56 \%$ of cases plinth was unprotected and had eroded in several locations. This included damage to the sandbags used for plinth protection. Given that in the rainy season mean total rainfall ranges from $307 \mathrm{~mm}$ in May to $733 \mathrm{~mm}$ in July (WMO 2020), this is a concern for the longevity of the shelters.

\subsubsection{Wall plates}

An important disaster risk reduction element is the effective tying of the wall plate and rafters to posts. This connection should use a tied and pegged fish mouth joint. Only $44 \%$ of shelters had the wall plates correctly tied to the bamboo posts. In general, all the ties were mostly observed to be of poor quality and often performed with the wrong rope, not $6 \mathrm{~mm}$ polypropylene for main connections. This might be the case due to the lack of the rope/wire provided, as mostly there was only one loop of wire or rope observed. Provision of enough rope and training on tying needs to be considered in future shelter intervention.

\subsubsection{Cross beams}

Cross beams absence was frequently observed. This is a concern due to the wind speeds in the area and because of the lateral forces on a structure that is not well anchored or braced. In addition, it was observed that several households had constructed lofts for storage, but with no cross beams to provide additional sturdiness to the structure. There is a potential for households to adapt the bamboo used to create 'loft' storage to form cross bracing without the need for additional material (see Fig. 6).

\subsubsection{Roof structure}

Rafters (bamboo which run down roof slope) are normally made from stronger elements than purlins (bamboo that run across the roof). Rafters, being the main support, also run under the purlins. In over $80 \%$ of shelters assessed both purlins and rafters were constructed using muli, rather than the rafters being of borak. Poor ties were observed in $80 \%$ of households. Especially the lack of tying down of rafters, this is a serious concern as the site is exposed to strong winds. Occasionally it was observed that purlins were 

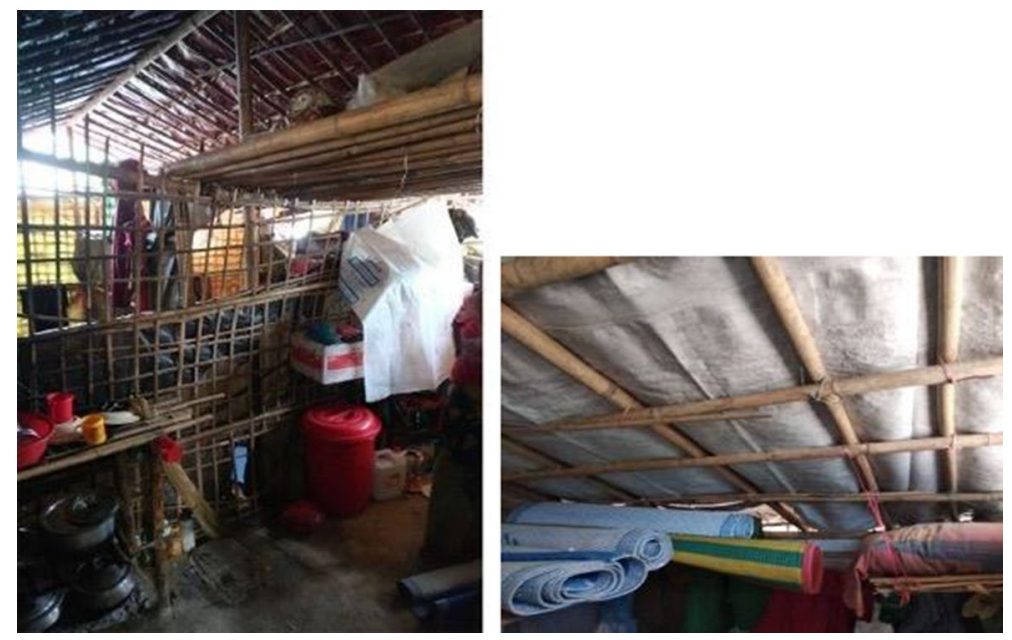

Fig. 6 Left: Use of additional bamboo to create a loft. If these members had been placed at 90 degrees and tied to the wall plates or posts, they would have also formed cross beams. Right: Rafter and purlins made of muli, with purlins placed under the rafters rather than on top

positioned under rafters and bottom purlins were tied to the wall plate rather than rafters (Fig. 6). 56\% households were identified with insufficient roof pitch ( $<20$ degrees).

\subsubsection{Bracing}

Bracing is key to disaster risk reduction. During the technical visits bracing was only observed in one shelter. Bracing was also identified as an element that was most frequently missing from upgraded shelters by FGDs with technical/field staff. During FGD the following were suggested as reasons: (i) Insufficient materials; (ii) 20-30\% of bamboo (muli and borak) provided was of inferior quality and hence could not be used; (iii) Lack of knowledge and understanding of importance of the bracing as well as the feeling that bracing takes additional living space; (iv) Households were in a hurry to complete the upgrades before the start of rainy season.

\subsubsection{Drainage}

Both drainage and retaining walls represent crucial disaster risk reduction components as they minimize the risk of flooding and landslides, which are a severe concern in the camps. Unfortunately, it was observed during the technical visits that $47 \%$ of shelters lacked any drainage. In $15 \%$ of cases it was mostly observed as draining directly on the path walls.

The following reasons behind lack of drainage (FGDs with technical/field staff) were identified: (i) an insufficient focus on household level drainage; (ii) insufficient space for drainage due to site congestions; (iii) drainage is being filled with sand/soil due to heavy rain. The following suggestion were made by the technical staff: (i) drainage needs to be designed according to hydrological models, including coordination with site management/ site improvement actors to ensure links to larger drainage channels; (ii) drainage channels need to be constructed with retaining walls; (iii) there is the need for regular follow up and maintenance; (iv) possible use of community based/led ownership for maintenance 
and small site improvements programs; (v) awareness program involving all age and gender groups as well as vulnerable community members so community can propose the best solutions.

3.3.7.1 State of shelter materials The state of the shelter materials was assessed during the technical visits to inform future interventions on the percentages of materials that could be reused or needs replacing. Concerns were voiced over the quality of the tarpaulin as well as the bamboo-both borak and muli (Fig. 7). Mostly bamboo damage was reported as being due to insects.

It was frequently observed that worn-out tarpaulin was replaced with black plastic polyethilene sheets. Inferior quality of tarpaulin was observed in $9 \%$ of tarpaulins used for walls and in $18 \%$ of tarpaulin used for roofing. Suggesting at least $30 \%$ of tarpaulin will need to be replaced. It is essential that tarpaulin for the roofing is of good quality.

The main concern with borak is borers infestation. In 38\% of households, $20-50 \%$ of borak had been infested with borers, while for $36 \%$ of household $50-100 \%$ was infested. While mould/rot, burn damage, and splitting was observed in less than $20 \%$ of households. More than half of borak bamboo will need to be replaced during the next phase of shelter interventions and this new bamboo needs to be treated before use.

During the technical visits approximately $30 \%$ of muli was found to be broken and/or split and needs to be replaced during the next six months. The broken and/or split muli can be reused for producing tiaras or woven bamboo walling (fence).

During the HLA, beneficiaries were asked how long they expect their shelters to last without future upgrades. 52\% of participants stated that their shelter will last for less than 3 months and no participant expected their shelter to last more than one year. The feedback provided is crucial in ensuring that the Sector further advocates for durable interventions. Proper quality control of procurement coupled with sufficient materials should be flagged.

3.3.7.2 Ventilation and air quality Insufficient ventilation was reported in the HLA, SSI and FGD. This was confirmed through the air quality measurements conducted by Bath university researchers. Poor ventilation is closely linked to poor air quality indoors (Beko et al. 2010) as well as an inability to keep indoor temperatures no higher than outdoor tem-

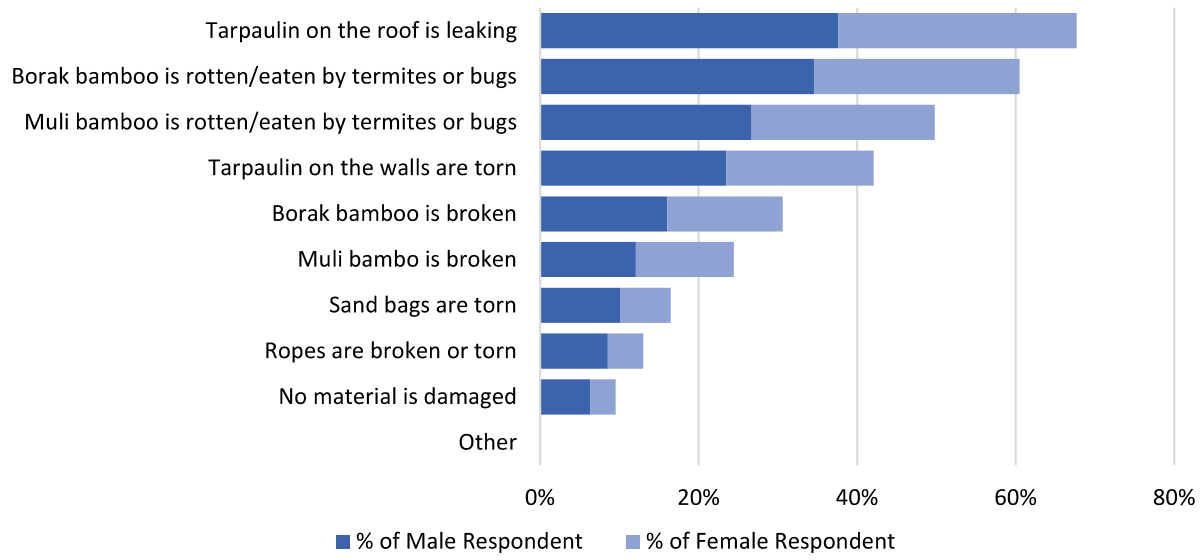

Fig. 7 Concerns over shelter materials 
peratures (Fosas et al. 2018). Refugees reported in the SSIs that they did not create openings in their shelters because of lack of materials and the difficulty of cutting a window in already weak structures. They also mentioned that the camp is very congested, with shelters built very close to each other, resulting in lack of airflow and sunlight. Consequently, most shelters are very dark, which is particularly challenging for women when they prepare food. However, light was not a priority for refugees, perhaps because monsoons and cyclones are their biggest worry, hence the structural safety preoccupies residents more than lack of natural light. Some people were simply lifting the tarpaulin up to provide some ventilation (see Fig. 8).

Smoke from cooking, and lack of ventilation pose health concerns in particular for the most vulnerable persons, such as infants, children, lactating and pregnant mothers, older, disabled and chronically ill persons (WHO 2010). During the HLA $16 \%$ of households reported that poor ventilation is one of the top 3 concerns (see Fig. 3). During the technical field visits $91 \%(\mathrm{n}=31)$ of shelters where perceived as having poor or no ventilation and $9 \%(n=3)$ reasonable ventilation. The majority $(62 \%)$ of shelters visited had no openings. In $20 \%$ of the shelters' openings were limited to the top of gable ends. Only one shelter visited had a window. The type of materials used appears to be contributing to this problem as using tarpaulin for both roofing and walling creates a near air-tight design. During FGDs some households suggested using woven bamboo wall cladding rather than tarpaulin to improve ventilation. When asked why openings have not been included in shelters for ventilation, beneficiaries frequently answered that they fear of theft and break ins. One of the households suggested raising their shelter so openings on the top of the walls would be higher.

As a result of insufficient ventilation, air quality was poor inside the shelters as revealed by the air quality monitoring. Data collected relating to particulate matter (PM) of sizes $1 \mu \mathrm{m}, 2.5 \mu \mathrm{m}, 4 \mu \mathrm{m}$ and $10 \mu \mathrm{m}$, commonly referred to as PM1, PM2.5, PM4 and PM10 is


Fig. 8 Examples of makeshift windows found in the camps 


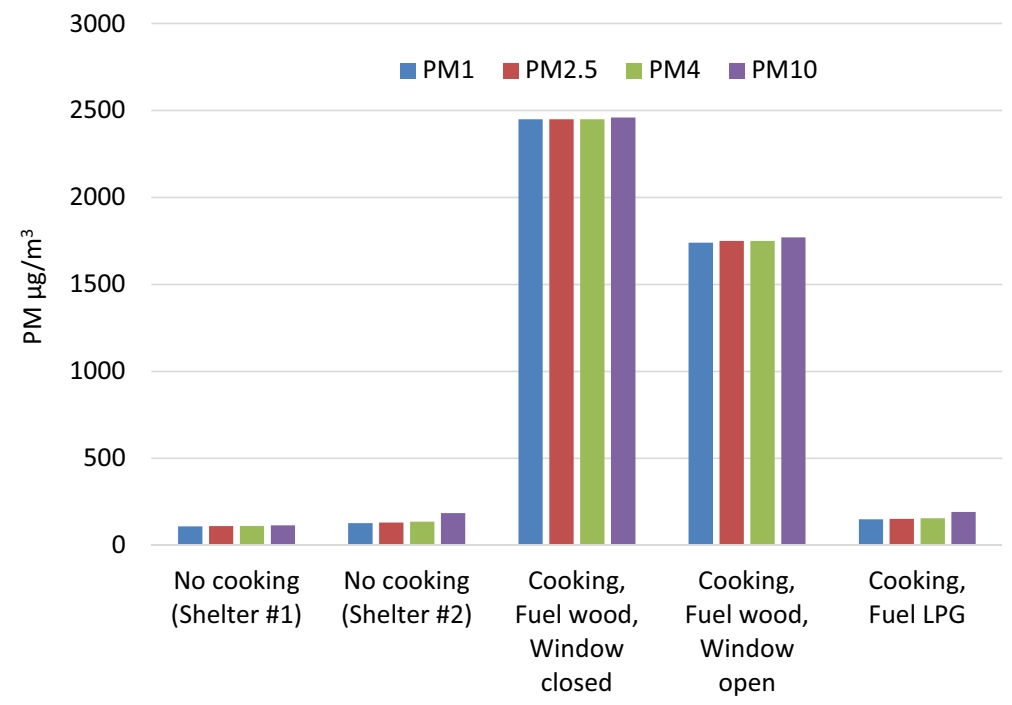

Fig. 9 Concentration of particulate matter (PM1, PM2.5, PM4 and PM10) in representative shelters measured over a 30-min sampling period

presented in Fig. 9. Under ambient conditions in the shelters when no cooking was taking place particulate levels between 109 and $185 \mu \mathrm{gm}^{-3}$ were measured. Kukadia and Upton (2019) summarise guideline concentrations for particulates from WHO and the EU Ambient Air Quality Directive and current UK Air Quality Standard (EU\&UK). The WHO guidelines recommend annual means of $20 \mu \mathrm{gm}^{-3}$ and $10 \mu \mathrm{gm}^{-3}$ for PM10 and PM2.5 respectively, whilst the EU\&UK suggest $24 \mathrm{~h}$ means of $50 \mu \mathrm{gm}^{-3}$ and $25 \mathrm{\mu gm}^{-3}$ for PM10 and PM2.5 respectively. Levels in shelters are clearly greater than those recommended as acceptable, even when no cooking is taking place. When cooking particulates (in the range

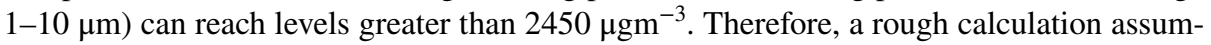
ing four hours of cooking per day suggests a mean in excess of $408 \mu \mathrm{gm}^{-3}$ or around 13 times the WHO limit.

Opening the windows in the shelter can help mitigate against the high particulate concentrations and reduce levels to below $1770 \mathrm{\mu gm}^{-3}$, however these concentrations remain of concern. The results show that an effective method of reducing particulates is to use LPG as an alternative fuel which lowered levels to that when no cooking is taking place. Total volatile organic compound (TVOC) concentrations were calculated as the sum of compounds eluting between (and including) n-hexane and n-hexadecane, quantified as toluene. Maximum TVOC levels inside the shelters when no cooking was taking place was $184 \mathrm{\mu gm}^{-3}$ and consisted of compounds including nonane, decane and undecane which are common solvents found in paints, adhesives and fuels, and decamethylcyclopentasiloxane which is an ingredient of personal care products such as antiperspirants hair care products. Levels of $8 \mathrm{\mu gm}^{-3}$ were measured during cooking with LPG and this particularly low value is attributed to variations between the different shelters that measurements were taken in. All these levels fell below the $300 \mathrm{\mu gm}^{-3}$ guideline concentration for VOCs suggested by UK building regulations. TVOC levels

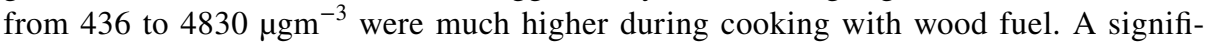
cant proportion of the VOCs consisted of benzene, up to $845 \mu \mathrm{gm}^{-3}$, and styrene up to 
$179 \mathrm{\mu gm}^{-3}$. These high levels are particularly concerning as these compounds are toxic and the WHO recommends no safe level for benzene and $70 \mu \mathrm{gm}^{-3}$ for styrene.

3.3.7.3 Thermal conditions The thermal conditions inside the shelters were monitored over a week. Figure 10 shows the time series in each shelter; Table 2 the percentage of time above the human comfort limit as given in Vellie et al. (2017). Clearly, all shelters are providing less than suitable conditions, with most shelters being above the comfort limit the majority of the time during active hours, thereby thermally stressing the occupants. The thermal stress is likely to be greater than indicated as many of the occupants are from vulnerable groups such as the elderly or very young.

It was noted by those completing the physical measurements that the underside of the roof tarpaulin was hot to the touch. This suggests that the air temperature measurements quoted above may not tell the whole story. In addition, the relative humidity of the location is high, with a mean of over $80 \%$ (internal and external) during the monitoring period. Hence, we have a combination of low ventilation and hence little air movement for evaporation from skin and clothing, high air temperatures and a high radiative component for the roof. It should be noted that one member of staff had to take a rest due to the heat in the shelters whilst making the measurements.

It was observed that some of the households had used blankets, jute bags or fabric to create a false ceiling, meanwhile others attached cardboard under the tarpaulin roofing in order to insulate the roof. Others had placed plant matter on the roof to shade the tarpaulin. As stated earlier, In the SSIs interviews $40 \%$ of refugees suggested improvements in thermal conditions as their top priority.

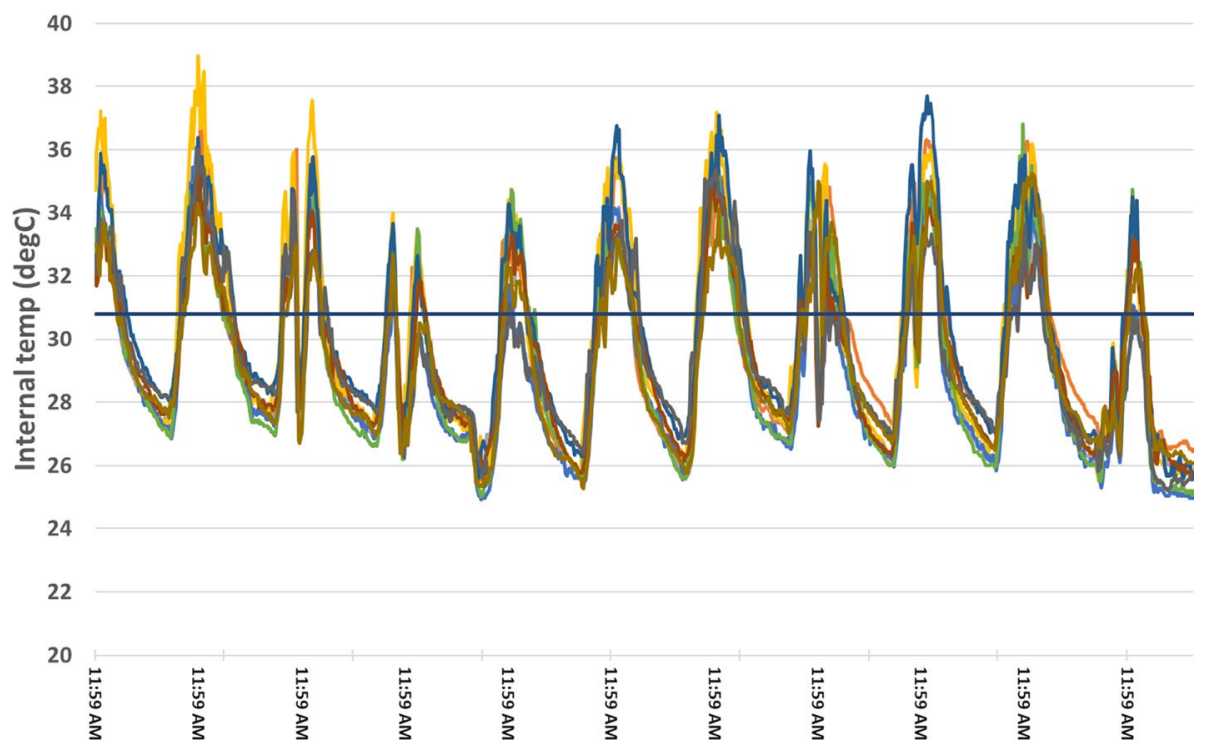

Fig. 10 Internal temperature time series in the nine shelters over 11 days. The horizontal line is the comfort limit, above which $80 \%$ of occupants will be thermally stressed 


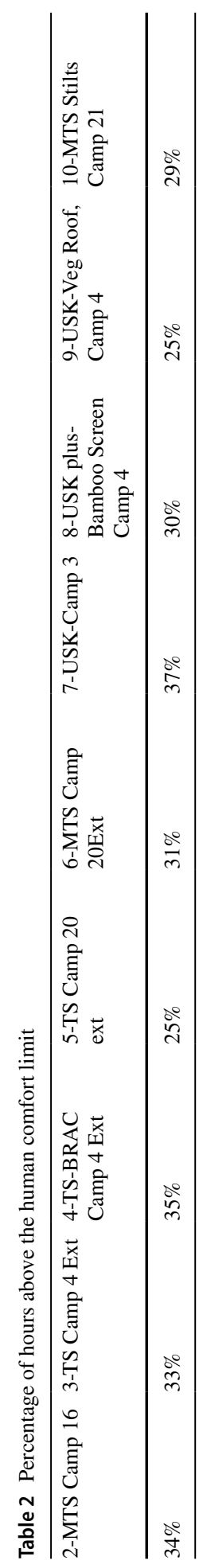


3.3.7.4 Privacy and security Privacy and security were addressed in all five elements of the study (HLA, SSI, technical field visits and FGD with households and staff). In the SSI the proximity of neighbours was found to cause concerns about privacy, especially for those refugees we spoke to who had lived in large homesteads in Myanmar, where houses were never built in close proximity. This has potentially contributed to the lack of windows. Therefore, when asked about their design preferences, it was thermal comfort, rather than privacy, that was the most important aspect of housing in the opinion of the refugees. Most people also reported having big verandas back home, and everyone interviewed wanted to have some private external space in the camps. In some camps however, verandas had been destroyed by "the authorities" as they were constructed without permission and were taking over already very limited public space, streets, path walks and drainages.

During the technical field visits of 34 shelters it was observed that $63 \%$ of shelters visited were attached to another shelter. The observation confirms that most households are living in some form of terraced shelters. This is a potential concern for protection and privacy. Of these terraced shelters, only one had a separation wall covering the complete height of the shelter. Separation and internal partition walls were mostly made from tarpaulin with muli support. $82 \%$ of the shelters visited during field visits had internal partitions (Fig. 11). Despite internal partitions, only 3 shelters included a closing door in the partition. In all other cases a curtain was used as a door, or the doorway left open. $80 \%$ of shelters could not be locked from the outside. However, the lack of a lock was only reported by $10 \%$ of respondents as being in their top three concerns during the HLA. Another concern related to security was also the type of wall cladding being used. During the technical field visits some households mentioned that they feared people cutting through their tarpaulin and entering their shelter. Having a bamboo tiara (bamboo lattice) on the outside of the wall would reduce this risk as bamboo is a tough material.

The SSI examined the problem of security in more detail. Refugees stated that at night they are afraid of child kidnappings and expressed concerns about supernatural beings. The interviewer explored these concerns with follow up questions, e.g. where do supernatural beings such as witches live, what do they look like, when do they visit you,
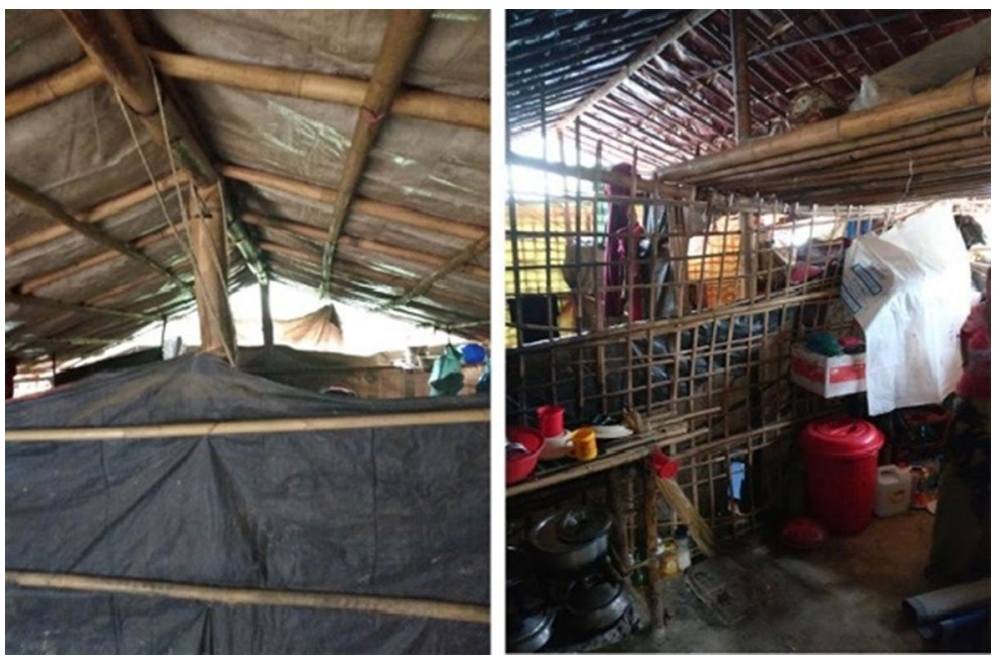

Fig. 11 Common separation between shelters (not internal partitions within a shelter) 
etc. It was crucial not to impose an etic lens, and not to disregard those beliefs as mere superstitions, when they have significant impact on people's health and well-being, in part because they influence behaviour.

Accusations of child kidnappings are a common occurrence in rural Bangladesh, sometimes leading to lynch mob violence. Our interlocutors suspected that kidnappers are Bangladeshis, but they did not think that their children were particularly targeted because of their origin. During the fieldwork in July 2019, there was a social media frenzy about child sacrifices surrounding the construction of the Padma bridge which started with Chinese sub-contractors using the phrase "that they needed more heads" ( sic) to complete the bridge. Discussions with staff exposed a folkloric belief linking construction projects with child sacrifices in Bangladesh, which dates back to the XIII century, when one of the rulers threatened to cut off their children's heads if they did not pay their feudal taxes. When asked why their children would be kidnapped, the refugees did not know the answer; possibly, apart from the folklore, it was also an expression of a general feeling that refugee camps were not a safe space for children. Some people also spoke of theft and attacks by terrorist groups as a concern, but very few experienced either of those.

Another aspect of beliefs noted related to a sense of (in)security is fear of malignant supernatural beings. The Rohingya believe that witches live by rivers, lakes and hills, all of which form the overall dominant landscape in the camps. Furthermore, witches are said to move into newly built shelters if they remain uninhabited. This has reportedly happened in camp 20 Extension which accommodates refugees whose shelters had been destroyed by landslides in other camps, as the dwellings built by shelter partners were empty for a few months awaiting the relocation of 80 families. As a result, witches were said to bother refugees at night, causing insomnia, diseases, including loss of eyesight and deafness, and mental health issues. This is part of strong animist beliefs and are part of oral and uncodified traditions, which co-exist with Islam in Myanmar, alongside more traditionally Islamic set of views concerning jinns, which are said to reside in unclean communal spaces such as toilets, as they are attracted to dirt. Unfortunately, there is a scarcity of ethnographic studies on religious practices of Rohingyas and due to time limitations in the field, we could not explore this issue further. What we learnt from refugees was that an imam would come to a hunted shelter and recite the Quran to warn off the evil spirits, however this does not always work: one man in camp 20 extension told us that two witches keep returning to his shelter even after the imam's prayers.

3.3.7.5 Activities: cooking and bathing As noted in the air quality measurements of PM and VOCs, cooking inside the shelters is a significant contributing factor for the poor air quality. Cooking on open fires in shelters is also a fire safety hazard that was voiced in both the FGDs and HLA.

During FGDs with households, lack of separate cooking space was identified as one of the main concerns. During the HLA it was observed that $18 \%$ of households listed attached/external kitchen as one of the three main improvements carried out with the shelter materials they had received. However only $6 \%$ of households suggested they would use materials received in the future to build an external kitchen. Given the poor air quality and lack of chimneys, this is a surprising result but can be linked to the overall lack of space; i.e. if there is more space available, the whole shelter would be expanded rather than the kitchen alone. Furthermore, people complained that their kitchen utensils are being stolen so they may have perceived external kitchens as even more prone to theft. 

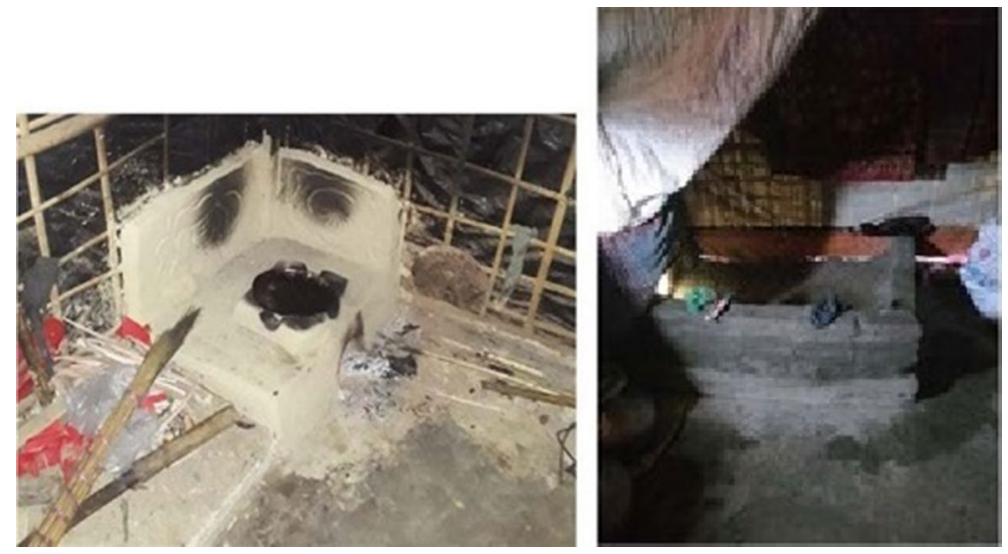

Fig. 12 left: Cooking area protected by small mud walls. The proximity of the flammable plastic tarpaulin and bamboo is evident. Right: Typical self-built in-shelter bathing area found

Out of all the households visited $88 \%$ had cooking taking place inside the shelter. Only in one case was a cooking area observed where flammable elements of the shelter were protected by CGI and in three cases with mud (Fig. 12). Given the materials used to construct the shelters, and the typical shelter spacing; this poses concern in terms of fire safety. Fire safety risks were voiced during FGDs with technical staff and 19\% of households listed it as one of the top three main concerns with their shelters.

The use of wood for cooking can cause additional issues. In 'Camp 20 extension' men reported walking a 12-h round trip to collect enough wood for 2 days of cooking.

As for bathing, it was noticed that beneficiaries are utilizing a portion of their shelter for bathing purposes (Fig. 12). Concerns related to bathing facilities were voiced during FGDs with households and listed as a shelter concern in HLA.

It was found that $40 \%$ of shelters had a bathing area. When people build their own shower rooms inside shelters, these are also used to urinate, with the waste exiting into the street/path. During the HLA $21 \%$ of households stated that absence of bathing facility in the shelter is one of their main shelter concerns. When asked what households would like to do with materials received in the future; $12 \%$ of them answered that they would build a private bathing facility.

\subsection{Assessment of Upgrade Shelter Kits (USK):}

\subsubsection{Training and technical assistance:}

Training and technical assistance forms an essential part any USK implementation. During the HLA it was found that $96 \%$ of households claimed to have received shelter materials, but only $76 \%$ of households recalled receiving training or technical assistance. Training/ technical assistance was perceived as either useful or very useful by $99 \%$ of households that recalled receiving it. Some of the participants also suggested that training could take a day and focus more on proper use of materials and how to make shelter stronger, as well as on risks such as high wind, flooding and landslides; and how they can be mitigated. When asked to specify three main things that they had learned during the training the following were frequently mentioned: (i) How to tie down shelter/roof; (ii) How to make anchor and 
stronger foundations; (iii) How to make strong ties and connections. The use of sandbags to create/protect plinth and bracing was also mentioned. When relevance of training was also discussed during FGDs with technical/field shelter staff. Topics that were mostly addressed were foundations and connections. All groups agreed that more practical sessions are needed and that training of trainers for partners should be longer than three days and it has been mentioned that training should be in Bangla rather than in English.

Regarding the training given to the beneficiaries, attendees agreed that female participation should be encouraged as they spend more time at home hence are more involved in day to day maintenance than men. Another suggestion coming from the technical/field staff during the FGDs was the importance of conducting follow up monitoring visits to ensure that the key messages have been adhered to.

\subsubsection{Materials}

Data was collected during HLA, FGDs with households and FGDs with technical/field staff. According to the FGDs with households and HLA the majority, 96\%, of households received materials that corresponded with the contents of the USK and/or the TDK.

Most households stated that they used USK materials to build a new shelter, enlarge the current shelter and/or build internal partitions (Fig. 13). In a few of the FGDs with households, tying down of the roof was also mentioned. These results appeared to be verified in the FGD with technical/field shelter staff where participants pointed out that most of the households constructed new shelters rather than upgraded emergency shelters. It appears that commonly USK materials were used to build new shelters and the old ones were torn down. With the materials (in this case ESK material) then reused to for enhancements of the new shelters built. This finding is significant for those selecting the materials that might be included in any USK.

In order to better understand how improve the USK the FGDs with technical/field shelter staff included the questions: (i) Do you see any issues with the USK? (ii) How could it be improved?

All groups agreed that the types of materials provided were insufficient especially as the kit did not include the elements required for bracing and internal partitioning. As households insisted on increasing the footprint of the shelter whenever possible, as such the

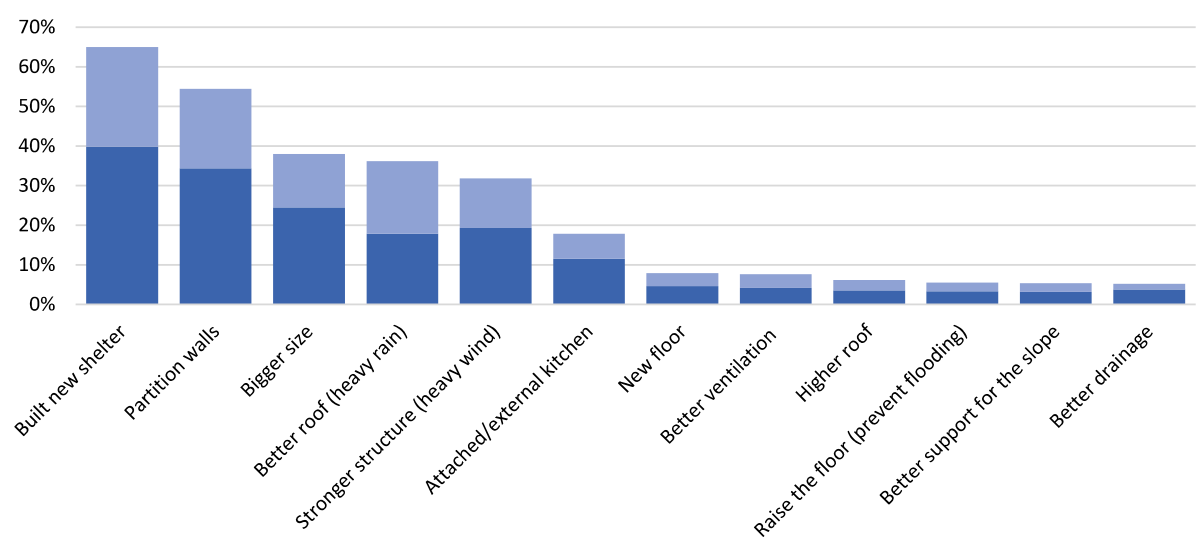

Fig. 13 The main improvements made with materials received-HLA 
volume of materials were insufficient. The poor quality of bamboo was also mentioned as one of the main concerns. Other suggestions were for the addition of two more borak per kit (thick bamboo used for structural purposes), 20 muli (i.e. non-structural thinner diameter bamboo), at least double the amount of rope, and one or two additional tarpaulins.

As the USK was used for both expanding the size and strengthening the existing shelters, unsurprisingly in all but one FGD with households, participants claimed to have purchased additional materials, with bamboo, tarpaulin and rope being the most reported items. Tarpaulins were used for repairs of damaged roofing and walling, as well as for internal partitions and extensions of the shelter. Similar answers were given during $\mathrm{HH}$ level assessment (Fig. 14) were approximately $20 \%$ of the participating households said that they purchased cement.

Given the volatile climate in Bangladesh and the hilly landscape, refugees are sometimes relocated to different parts of the camps due to landslides and floods. The SSIs indicated that occupants believed the bamboo and tarpaulin were too weak to resist heavy rain falls and wind. They also thought that the materials provided were insufficient to make the dwellings more robust. When asked about what adaptations they would do if they had the resources, all people interviewed expressed their wish to 'strengthen' their shelters.

The use of cement was frequently observed during field visits where many shelters had a thin layer of cement mixture covering the floor. According to the HLA, 22\% of households claimed not to have purchased any materials, although it is important to note that some of households might have been unwilling to admit to purchasing materials in fear of not receiving assistance in the future.

\subsection{Materials desired and future improvements}

One of the main purposes of the work was to inform future shelter interventions including the type and quantity of materials needed. During FGDs with household participants

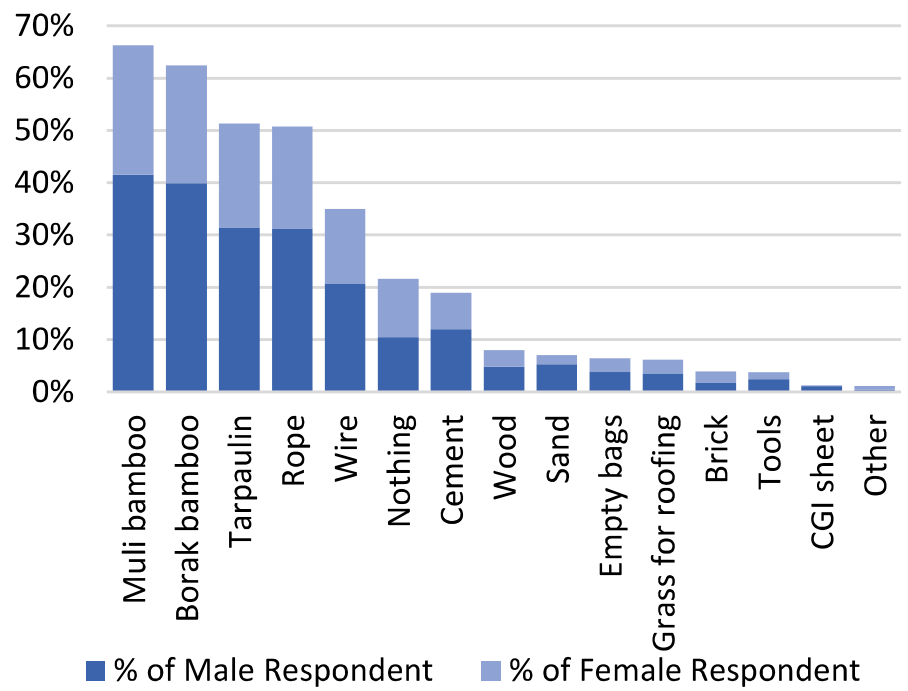

Fig. 14 Materials purchased by households-HLA 
Materials households would like to receive for futre upgrades

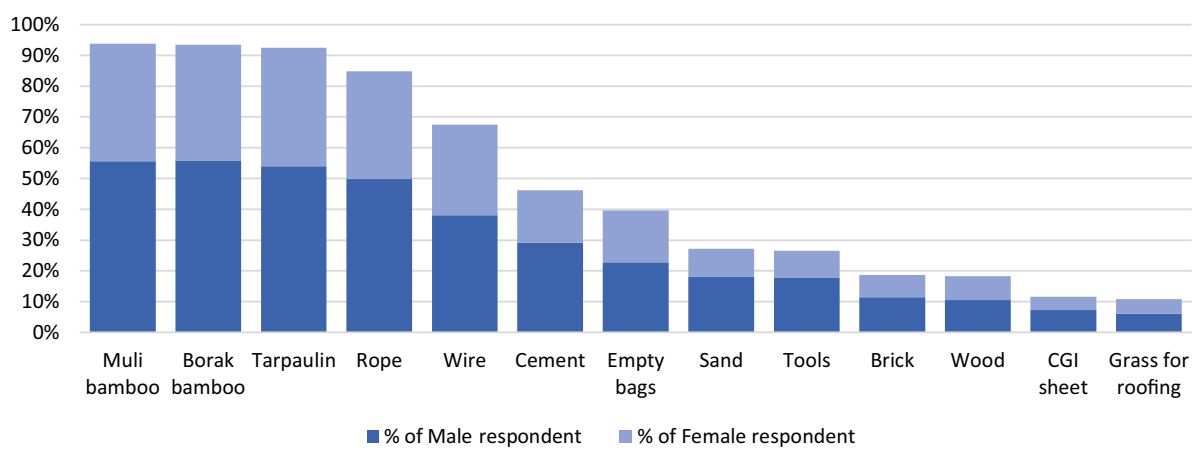

Fig. 15 Materials households would like to receive during future upgrades-HLA

mostly listed bamboo (borak and muli) and tarpaulin followed by rope and wire as desired materials. They also mentioned bamboo fencing (i.e. woven bamboo walling) and sandbags (Fig. 15). HLA results were similar to the FGDs. During the HLA participants were also asked about the respective quantity of each material needed.

With respect to materials currently supplied, several of participants mentioned that they would like to have shelters similar to their homes in Myanmar, i.e. a wooden house with wooden posts and straw or CGI (corrugated galvanised iron/steel) roofing. Brick for walls was mentioned as a desirable alternative as were reinforced concrete (RC) posts.

Fifty-two percent of households identified cement as needed during the household level assessment. Cement would not be used for construction of the walls but rather for the cement flooring and potentially for creation of bathing area in the shelter. Cemented flooring may have positive effect on the overall level of sanitation and hygiene (for the bathing areas in particular) as it protect from potential bugs and vector borne diseases (e.g. sand-flies, scabies). Hence the provision of cement for flooring and bathing facilities might be further explored as well as concrete foundations and posts in the next round of shelter interventions.

The provision of brick can be considered for the creation of non-flammable kitchen area or bathing areas in the shelter. Brick walling is unlikely to be possible as it indicates permanence and hence is unacceptable by the Bangladeshi government. The issue around permanence is a political one and therefore what materials are regarded as permanent has no relation to the properties of the material but rather their visibility. For the roof, grass/straw should be considered as a material for future interventions as it provides better insulation to the shelter then tarpaulin roofing and has 2-3 years' life span, which is more than tarpaulin. Twenty-one percent of households requested 1-2 sheets of CGI, which indicates that they do not intend to use it for roofing but rather for doors (some CGI doors were already observed in the camps). CGI is banned for all shelter construction as it can present significant hazard during the high winds.

\section{Discussion}

Each of the approaches used by the transdisciplinary team exposed different issues, from the poor state of construction materials, to the impact of the belief in witches. 
Based on the main shelter concerns expressed by households and technical/field staff it is clear that the primary concerns of the affected community are material quality; and ensuring the strength of their shelter. This was somewhat already known to the shelter aid sector and partners working in the camps as the main intent of distributing USK was to strengthen the shelters. However, lack of space or high density was also reported as a main concern in the FGD and therefore materials provided were used to expand shelters rather than strengthen them. As such, it seems that although concerns related to "quality of materials" and "strength of shelter" rank higher than concerns about privacy and security, they are on par with space.

By a way of comparison, research done in refugee camps in Jordan where shelters were agency-provided rather than self-built (Albadra et al. 2017) the issue of structural safety was not raised, and security and privacy ranked as very high. This does not mean that privacy and security should not be seen as important issues for the Rohingya refugees in Bangladesh, but that shelter strength and durability of materials need to be prioritised in self-built shelters in this geographically difficult context.

As for overcrowding and space, with the majority of households containing more than 4 members it would appear advisable for the humanitarian sector to consider 5 household members as the baseline for shelter sizing rather than four. The covered living space per person is below the common Sphere Indicator of $3.5 \mathrm{~m} 2 /$ person for many families. In accordance with Sphere Guidance Note 3 on Covered Living Space (Sphere 2018): "further consultations should be undertaken with the affected community, including members of vulnerable groups and those caring for such individuals, regarding existing local practices in the use of covered living space, for example sleeping arrangements and the accommodation of extended family members', to determine if the standard itself is being met. The protracted nature of the displacement makes this appear unlikely as the standard was only intended for the initial emergency phase of response. Linking the findings on covered living space with the population density in the camps shows that in most cases households also have little or no space to extend their shelter. It is likely that ensuring sufficient covered living space is mostly limited by lack of space, rather than the lack of materials. Discussion with affected communities should be prioritized to determine effective ways to alleviate the stress that this overcrowding is creating, while continuing to pursue opportunities for decongestion.

The fact that most of the shelters are attached to other shelters will need to be considered when providing future shelter interventions. Traditional houses in Myanmar had verandas. Therefore, these were desired by the refugees as they help with privacy and escaping the heat of the shelters. Further discussion should be conducted with households regarding the potential inclusion of additional materials for partitioning walls that will reach to the apex of the roof and provide additional privacy if needed. This may also need to be taken into consideration when upgrading the strength of shelters through the addition of roof plane bracing or other features that may be necessary in larger/ longer structures. For most families the amount of materials supplied was not considered sufficient. Most of them chose to buy additional materials, generally in the same form as those being provided by agencies, while a small number of households purchased alternative more durable materials. This may indicate that USK material choice closely matched needs or it might indicate lack of alternative materials in the markets that can be accessed. Future shelter interventions will need to allow for much more freedom of choice regarding materials received. Furthermore, the quality of materials with bamboo either rotting or infected with termite was revealed as a major issue. 
Additionally, ventilation in terraced shelters may need to be addressed as end of roof venting option may be more limited than in individual shelters. Terraced shelters also pose an additional risk for spread of flames due to the absence of fire-breakers.

As a result of using different methods to collect data in this research, some of the results may seem contradictory. For example, participants were least satisfied with thermal comfort as reported in the SSI and it was reported as their top priority. However, during the FGD and HLA, poor ventilation and high temperatures in shelters were considered as a problem but they did not appear to be prioritizing it above the other shelter improvements. Potentially because people had no capacity to improve it due to the lack of a viable alternative, lack of materials, money or knowledge. To address this concern, it would be beneficial to carry out additional FGDs with households and an added focus on women, older and disabled persons, who may spend considerably more time inside shelters, to further discuss and learn how ventilation and heat in the shelter can be addressed in a culturally appropriate and affordable way.

Cooking in shelters presents a major concern especially as the cooking area is in most of the cases is not protected by non-flammable material. Protecting the cooking area with a mud wall has already been observed in the camps and it could be one of the alternatives to be explored further in discussion with communities when developing future shelter interventions. The air quality measurements show the presence of high levels of toxic compounds (VOCs) and that PM levels in shelters are significantly higher than recommended levels. When cooking particulates can reach levels greater

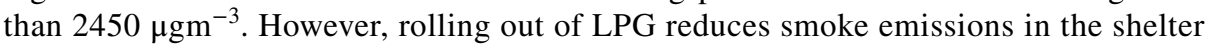
but the fire hazard will remain. Our findings are in line with other studies in refugee camps in Ethiopia where ethanol stoves were used to replace solid fuel burning for cooking; it was found that the average PM2.5 concentrations over $24 \mathrm{~h}$ for the 33

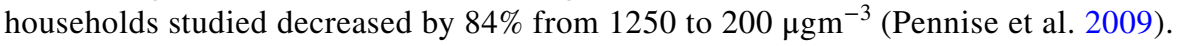

Household level bathing facilities provide the necessary privacy and security to beneficiaries especially women as such should be considered in the future shelter interventions. As this is a multisectoral issue that related to drainage and hygiene it would be advisable to further assess solutions with other aid sectors.

\subsection{Impact of this work}

As a result of the findings of this work, Phase 3 of shelters upgrades was rolled out in 2019 and early 2020 with new shelters interventions known as 'Transitional Shelter Assistance'. This type of assistance was developed based on the lessons learnt from this work with material durability, space, tailored training, strength of shelter, and freedom of choice at the core of this approach. Currently over 70,000 households benefited from transitional shelter assistance. Where possible households were relocated to allow for $3.5 \mathrm{~m} 2 /$ person. Poor or damaged building materials were replaced with more durable options, especially treated bamboo and footings. Households were also supplied with additional materials, technical assistance and targeted training. Furthermore, LPG (liquid propane gas) have been (and continue to be) distributed for cooking in order to reduce the health risk due to poor air quality. The success of these new interventions is currently being assessed. 


\section{Summary and conclusion}

The majority of shelter studies rely on a very limited array of methods and skillsets. Here we have used a transdisciplinary team and a wide set of methods to see if the different approaches draw out different findings, or the same. The work was based within the world's largest refugee camp (UNHCR 2019) and had a sample size of 1594 families.

The work included a household level assessment, semi-structured interviews with households, technical field visits, separate focus group discussions with beneficiaries and technical staff, physical measurements of temperature and air quality, and satellite imagery. The team included general humanitarian staff, agency engineering staff, building physicists and an anthropologist. Each method helped reveal different issues: (i) the satellite imagery that a third of the camp population is housed below the $3.5 \mathrm{~m}^{2}$ per person target, indicating overcrowding; (ii) the technical field visits that the majority (63\%) of households were living in terraced shelters with little separation/privacy between households, and that shelter upgrade kits designed to strengthen shelters against extreme weather events had mostly been used to expand shelter size; (iii) although the household level assessments uncovered the issue of poor ventilation, it was only the semistructured interviews that exposed the reasons behind it (difficulty of cutting openings whilst maintaining structural integrity and ease of opening and closing any openings), and only the physical measurements that could assess the implications of this-annual particulate exposure 13 times the WHO limit, particulates reaching levels greater than $2450 \mu \mathrm{gm}^{-3}$, the existence of benzene $\left(845 \mu \mathrm{gm}^{-3}\right.$ maximum $)$ and styrene $\left(179 \mu \mathrm{gm}^{-3}\right.$ maximum). (iv) The focus groups identified internal temperatures to be an issue, but only the physical measurements could identify the scale of the issue, with most shelters over the comfort limits for the majority of daylight hours; (v) Security was revealed as an issue by various methods, but only the more freeform semi-structured interviews exposed the reasons behind concerns over child kidnapping and beliefs about witches. (vi) The household level assessments and focus groups dedicated to the occupants pointed to issues with material shortages and poor quality materials, but only the focus groups with the technical team exposed the need for additional training, including gender-sensitive technical training tailored for women on the correct use of the materials.

From this work it is clear that a diverse team using a multitude of approaches to data gathering and working in a transdisciplinary manner has much to offer the sector, as not only does it successfully expose issues, it can discover the reasons behind them, and, by including quantitative physical measurements allows costed improvement plans to be developed, targets to be set and general, rather than case specific, knowledge to be generated. The impact of this collaborative work is already being observed with the role out of new shelter assistance and LPG stoves across the camp. 
Acknowledgements The University of Bath would also like to thank IOM for the support to secure the required access approvals to the camps and the availability of the Shelter and NFI resources. The authors acknowledge the following organisations' contribution to the collection of data and facilitating this research: Action Contre la Faim (ACF), Bangladesh Rural Advancement Committee (BRAC), CARE, Caritas Bangladesh, Catholic Relief Services (CRS), Christian Aid, Danish Refugee Council, International Organization for Migration (IOM), PULSE/People in Need (PIN), RISDA, Save the Children, Solidarités, United Nations High Commissioner for Refugees (UNHCR), World Concern /Medair. In addition to the help of Andy Dengel, Keith Mower and Carla Da Silva from BRE Watford, for air quality analysis of VOCs. Part of this research was funded by EPSRC/GCRF (EP/P029175/1). Appendices and the data collected during this research is openly available at the University of Bath Research Data Archive [https://doi.org/10.15125/ BATH-00781].

Open Access This article is licensed under a Creative Commons Attribution 4.0 International License, which permits use, sharing, adaptation, distribution and reproduction in any medium or format, as long as you give appropriate credit to the original author(s) and the source, provide a link to the Creative Commons licence, and indicate if changes were made. The images or other third party material in this article are included in the article's Creative Commons licence, unless indicated otherwise in a credit line to the material. If material is not included in the article's Creative Commons licence and your intended use is not permitted by statutory regulation or exceeds the permitted use, you will need to obtain permission directly from the copyright holder. To view a copy of this licence, visit http://creativecommons.org/licenses/by/4.0/.

\section{Appendix 1: The kits}

See Table 3.

Table 3 Content of USK and content of TDK

\begin{tabular}{lllll}
\hline Content of USK & & & Content of TDK & \\
\cline { 1 - 1 } \cline { 5 - 5 } Item & Amount & & Item & Amount \\
\hline Tarpaulin & $2 \mathrm{pcs}$ & & $6-8 \mathrm{~mm}$ Rope & $60 \mathrm{~m}$ \\
Borak bamboo & $4 \mathrm{pcs}$ & & Steel peg or & $6 \mathrm{pcs}$ \\
Muli bamboo & $60 \mathrm{pcs}$ & & Sand bag & $10 \mathrm{pcs}$ \\
$6 \mathrm{~mm}$ rope & $1 \mathrm{~kg}$ & & & \\
$3 \mathrm{~mm}$ rope & $1 \mathrm{~kg}$ & & \\
GI Wire & $0.5 \mathrm{~kg}$ & & \\
\hline
\end{tabular}




\section{A. Houshold information ( খানার তথ্য)}

A1. Gender of the head of the household ( খানার প্রধान পুরুষ নा মशिলा ?)
Male পুদুপ
Female মशिला
Other अन्यान्य উज্লেখ कळ्रन

A2. Age of the head of the houshold ( থाना প্রধाনের বয়স কত ?)

A3. Is the person responding the head of the household (উত্তরদাতা কি পরিবার প্রধান ?)

Yes श्गाँ

No ना

A3.1. Gender of the person responding (উত্তরদগাতা कि পুরুষ নा মহিলা ?)
Male পুরুষ
Female मरिला
Other অन्यान्य উज্्েখ কর্নু

A3.2. Age of the person responding (উত্তরদাতার বয়স কত?)

A4. Total number of MALES in the household (খানায় মোট পুরুষের সং খ্যা কত?)

A5. Total number of FEMALES in the household (খান্য় মোট নার্রীর সং খ্যা কত?)

A6. Total number of $\mathrm{HH}$ members is NaN (খানায় মোট সमস্য সং খ্যা কত?)

\section{B. Current shelter বৃত্মান শেল্টার}

B1. Did your household recieve any materials to upgrade/construct your shelter ( আপনার পরিবার কি শেল্টার উন্নত/মজবুত করে তৈরীর জন্য নির্মণ সামগ্রী পেয়েছে?)

Yes शाँ

No ना

I don't know आयि জानि ना

B2. How long ago did your household receive the most recent shelter materials? ( আপনার পরিবার্ন কতদিন আগে আপনার এ শেল্টার্র তৈর্রীর জন্য মালামাল পেয়েছেন?)

\section{Before 2018 ২০১৮ সালের আগে}

in January or February জানুয়ারী বा ফেন্বূর্য়ারিতে

in March or April মার্চ বा এপ্রিল মাসে

in May or June মে বা জুন মাডে

less then a month ago এক মাসের ও কম সময় আগে

Do not remember মনে করতে পারছি না 


\section{Appendix 2: Household level assessment questionnaire}

B3. Did your household receive technical assistance/training on how to use construction materials to upgrade your shelter? ( কিভাবে নির্মাণ সামগ্রী ব্যবহার করে শেল্টার মজবুত করে তৈরীী করতেত হয় জে বিষয়ে আপনার় পরিবার কি কারিগরী नহায়তা/প্রশিক্ষণ (েয়েছেন?)

yes we received technical assistance হ্যা, আমরা কারিগরি সহযোগিতা পেয়েছি।

$\square$ yes we received traning হ্যা, আমরা প্রশিক্ষণ পেয়েছি।

No we did not recieve technical assistance or training না, আমরা করি গরি সহযোগিতা বা প্রশিক্ষণ পাইনি

I don't remember মনে করতে পারছি না

B3.1 How usefull was technical assistance/training that you received? আপনি যে কারিিগরি সহয়তা পেয়েছেন, সেটা কতটা काज़ ছিল?

( very useful थूবই काख़র

Useful काজর

Not useful काজর নয়

B4. What are the 3 main improvements you made with materials received? (ফে মালামাল আপনি পেয়েছেন তা দিয়ে আপনার্র শেল্টার মজবুত/উন্নত করেছেন এমন ৩টি কি প্রধান গুরুত্বপূর্ণ কাজ কি কি?)

please select 3 ৩ সिलनख্ট करन

Built new sheter নতুন শেল্টার তৈরী

Bigger size তুলनाমূनক বड़ Mाकाর

Partition walls পাটিশন দেয়াল

Attached/external kitchen সং যুক্ত / বাইबের রান্ধাঘর

New floor নতুন মেবো

Stronger structure to resist heavy wind क্রারী বায় প্রতিরোধ করার জন্য শন্তিশালী গঠনের শেন্টার

Better roof to resist heavy rain ভात्री বৃষ्दि প্রতির্রোধের জন্য ভাল ছাদ

Better ventilation to resist heat (openwondows or/and doors) তাপ প্রতিরোধ করার জন্য ভাল বাযুচলাচল (৫োলা জানালা বা / গবः দর্তা

Higher roof উচ্চ ছাদ

Better drainage ভाल निक्षाশन

Better support for the slope চाল़র ঢারের জন্য ভাল সমর্থন

Raise the floor/used the sandbags to prevent flooding বন্যা প্রতিরোধ করার জন্য শেন্টারের রেবা উঁচু করুন/বানির বন্তা ব্যহার করুন

Nothing किं ना

Other অন্যান্য উডল্লেখ করুন

B4.1. If other - specify ( অन्্যান্য হরে नির্দিষ্ট কর্নে উল্লেখ কণুন) 


\section{B5. What do you see as the main issue with your shelter (list top 3)}

$\square$ Foundations are not strong ফাউযেশনগুলি শক্তিশালী নয়

$\square$ Walls are not strong দেয়ালগুলি শক্তিশালী নয়

Walls are not good - wind/rain is coming through দেয়ালগুলি ভাল নয় - বाয় / বৃষ্টির মধ্য দিয়ে আসছে

$\square$ Roof is not well built ছाम जान नड़

$\square$ Roof is damaged श्ञा कতিগ্রস্ত

$\square$ Ventilation in the shelter is bad আশ্রয় এর মখ্যে বায়ুচলাঢল খারাপ

$\square$ Floor is bad quality घরের তল/মেবা খারাপ মানের

$\square$ Materials are damaged/rotten সামগ্রী ক্ষতিগ্রন্ত / পচে গেছে

$\square$ Shelter gets flooded/wet when it rains घथন বৃষ্টি হয় তথন শেল্টার প্লাবিত হয় / ভিজা হয়

$\square$ Shelter is nat tied down শেল্টারকে টেনে মাটির সাথে বাঁধा হয়নি

$\square$ There is no bathing facility in shelter শেল্টার এ গোসল এর কোন ব্যবস্থা নেই।

Shelters are too close together (risk of fire) শেল্টার গুলো থুব কাছাকাছি অবস্থিত (আগুন লাগার বুঁকি)

$\square$ Not safe (no locks, risk of break in) সুরक्্िত নয় (তালা নেই, তে তে তোকার ঝুঁকি)

$\square$ No privacy for women and girls নারী ও মেয়ে দের জন্য গোপনীয়তা নেই

$\square$ Other অन्यान्य উज্লে কक्न

$\square$ Nothing किषू ना

B5.1. If other - specify ( অন্যান্য হরে নির্দিষ্ট করে উল্লেখ করুন)

B6. Is any material in your shelter broken or damaged? ( এখন আপনার মরের কোন উপাদান কি ভাঙ্গা আছে বা নট হয়েছে? )

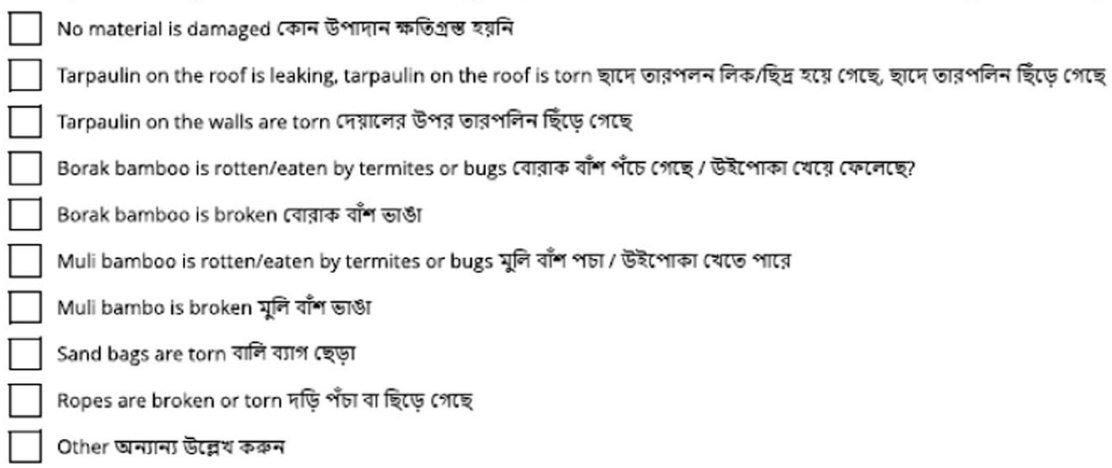




\section{Future upgrade (ভবিষ্যতে মর মজবুত করা)}

C1. What shelter materials would you like to receive to improve your shelter? শেল্টার এর উন্নয়নের জন্য আপনার কি কি जिनिम लाগবে?

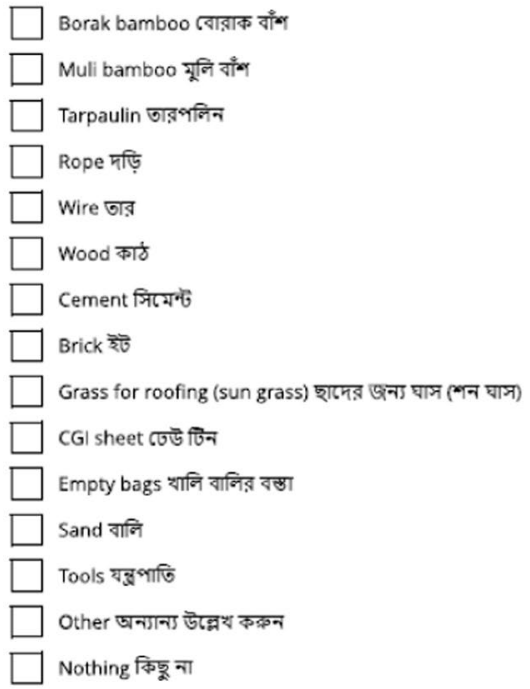

C1.1. If other- specify ( অन्यान्य হলে নির্দিষ্ট করে উল্লেখ কন্পুন)

C2. What would you use materials for (Select top 3)? আপনি জিনিস ওুলো দিয়ে কি করববেন (প্রেথম ৩ টি বলুন)?
$\square$ Build new house नতून বाসा তৈরি
$\square$ Replace bamboo বাঁ বদল ক़ুন
$\square$ Replace tarpaulin তার্পলিন বদল করযেন
$\square$ Make better foundations जान ভিত্তি তৈরি কর়েেন
$\square$ Make house stronger (bracing) घর শক্কিশালী করবেন ( ব্রেসিং)
$\square$ improve the roof ছাদ উন্নত করবেন
$\square$ Increase the size of the house বাড়ির আকার বাড়াবেন
$\square$ Change the materials of walls দেয়ালের সামগ্রী/উপকরণণ পরিিবর্তন করবেন
$\square$ Increase/improve ventilation বায়ু সঞ্ঞালন २६্টি বा উন্নয়ন
$\square$ Add internal partition অভ্যন্তরীর পাটিশন যোগ করযেন
$\square$ Build private bathing facility ব্যাক্টিগত গোসলখানা তৈরি
$\square$ Build external kitchen বাইরে রান্মাঘর তৈরি
$\square$ Other অन्यान्य উল্লে কकন
$\square$ NONE কোনটাই नয়

C2.1. If other - specify ( অন্যান্য হলে নির্দিষ্ট করে উল্লেখ কর্যুন) 


\section{Appendix 3: Semi-structured interviews questionnaire}

Section 1: Background info about the participant and shelter:

Number of people living in the shelter:

Size:

Ask/observe: General description of original shelter; materials, amenities available etc

Ask/observe: General description of adaptation made:

Why have you made these adaptations? i.e. establish motives

What other adaptations would you like to make to your shelter if you could?

\section{Section 2: Design questionnaire: About your shelter}

1- How satisfied are you with the PRIVACY your shelter provides?

Satisfied - neither - unsatisfied

Why?

2- How satisfied are you with the STRUCTURAL SAFETY i.e. fire risk, strength of construction your shelter provides?

Satisfied - neither - unsatisfied

Why?

3- How satisfied are you with the amount of SPACE your shelter provides? i.e. is it big enough?

Satisfied - neither - unsatisfied

Why?

4- How satisfied are you with the light levels in your shelter?

Satisfied - neither - unsatisfied

Why?

5- How satisfied are with the protection your shelter provides against wind and rain?

Satisfied - neither - unsatisfied

Why?

6- How satisfied are you with the thermal conditions in your shelter?

In summer (rainy season): 
Satisfied - neither - unsatisfied

Why?

In winter (dry season):

Satisfied - neither - unsatisfied Why?

7- How satisfied are you with the air quality of your shelter?

Satisfied - neither - unsatisfied Why?

8- Do you feel comfortable receiving guests in your shelter?

Yes - no

Why?

9- Is it possible to change/adapt the shelter to suit your needs?

Yes - no

Why?

10- Where do you cook? Are you happy with kitchen facilities?

Yes - no

Why?

11- Where is your bathroom? Are you happy with bathroom facilities?

Yes - no

Why?

12- Do you feel safe inside your shelter during the day?

Yes - no

Why?

13- Do you feel safe inside your shelter during the night?

Yes - no

Why?

\section{Section 3: Design questionnaire: your thoughts on a better shelter}

14- Please rank the following aspect of design in order of importance:

\begin{tabular}{|l|l|}
\hline Appearance (form and material) & \\
\hline Flexibility/ adaptability (e.g. to change layout of shelter) & \\
\hline Security & \\
\hline Privacy & \\
\hline Thermal Conditions & \\
\hline Space (is there enough space for your family and guests) & \\
\hline Private Bathroom & \\
\hline Private Kitchen & \\
\hline Air quality & \\
\hline Lighting Level & \\
\hline
\end{tabular}

15-Could you tell us a little bit about your house in your country of origin; what was it made of; size etc

16-Discuss any aspects that the participant would like to discuss in further detail. 


\section{Appendix 4: Focus group discussions with technical staff}

\begin{tabular}{|c|c|}
\hline \multicolumn{2}{|l|}{ FGD Planning } \\
\hline Title & Shelter assessment - Field engineers, Shelter Field Officers \\
\hline Target group & $\begin{array}{l}\text { Selection criteria } \\
\text { 1) Engineers and Shelter Field Staff working in camps (KTP, Teknaf, Shamlapur, Chackmarkul and Unchiparang) } \\
\text { 3) 2-3 members for each partner willing to participate } \\
\text { 4) } 8 \text { people per FGD (1 FGD to be done in Teknaf) } \\
\text { Preferable diversity } \\
\text { 1) Including female staff }\end{array}$ \\
\hline Objectives & $\begin{array}{l}\text { 1) To assess Shelter technical staff perception on what materials and assistance are most needed/relevant for the } \\
\text { next phase } \\
\text { 2) To assess Shelter technical staff perception towards durability of current shelters } \\
\text { 3) To gather Shelter technical staff opinion on alternative materials and mud walls. } \\
\text { 4) To gather Shelter technical staff observation on the main shelter issues }\end{array}$ \\
\hline Brief & $\begin{array}{l}\text { Presenting the team members and explaining that this FGD is part of a study to assess most pressing needs in terms } \\
\text { of construction materials to improve shelters. } \\
\text { We are interested in better understanding how families coped with the situation, to identify the main challenges } \\
\text { and to listen to your opinion. We would also like to learn about suggestions on alternative materials and } \\
\text { techniques. } \\
\text { Answers are anonymous. FGD will be in Bangla. }\end{array}$ \\
\hline Questions & $\begin{array}{l}\text { 1. Based on your field observations, how many households sufficiently upgraded their shelters? (What did } \\
\text { the majority of HH do with the materials received? What were the elements that were most frequently } \\
\text { missing from the upgrades? } \\
\text { 2. Do you see any issues with USK? (Insufficient materials, insufficient training...). How could it be } \\
\text { improved? } \\
\text { 3. What are the major issues that you see with the current shelter? (not strong enough, materials are not } \\
\text { durable, shelter are too small, lack of ventilation, shelters are flooding, USK were not properly used...) } \\
\text { 4. What would you suggest to improve current shelters? (Construct shelters, provide more materials, } \\
\text { provide more durable materials, more training, cash to HHs for materials...) } \\
\text { How many materials that were given to the households as USK do you think can be reused if additional } \\
\text { 6. What materials and how many do you suggest to provide to households to make their shelter more } \\
\text { 7. What about any materials that were not provided? (cement, brick) Or alternative building techniques } \\
\text { (wattle and daub, mud walls...) } \\
\text { 8. Do you think that technical assistance/training provided to households is sufficient/relevant? (how can it } \\
\text { 9. Do improved?) } \\
\text { 10. Do families using the shelter face any issues (lack of privacy, poor ventilation...) How do you think this } \\
\text { 11. Do you have any discussion with the households? What do they tell you about their shelter? }\end{array}$ \\
\hline
\end{tabular}




\section{Appendix 5: Focus group discussions with households}

\begin{tabular}{|c|c|}
\hline \multicolumn{2}{|c|}{ Household Level FGD } \\
\hline Title & Shelter Survey - FGD for Roghingya Community \\
\hline $\begin{array}{l}\text { Target } \\
\text { group }\end{array}$ & $\begin{array}{l}\text { Selection criteria } \\
\text { 1) Refugees living in camps (KTP, Teknaf, Shamlapur, Chackmarkul and Unchiparang) } \\
\text { 3) Male } 1 F G D \text {, Female } 1 F G D \text { or Male EVI 1FGD, Female EVI 1FGD } \\
\text { 4) 8-15 people per FGD } \\
\text { Preferable diversity } \\
\text { 1) Refugees coming from different camps } \\
\text { 2) Some refugees with construction expertise } \\
\text { 3) Ensure wide age representation }\end{array}$ \\
\hline Objectives & $\begin{array}{l}\text { 1) To assess community perception on durability of shelters } \\
\text { 2) To assess community perception on what materials and assistance are most } \\
\text { needed/relevant for the next phase } \\
\text { 3) To assess community perception toward mud walls and other alternatives }\end{array}$ \\
\hline $\begin{array}{l}\text { Facilitator } \\
\text { and note- } \\
\text { take roles }\end{array}$ & $\begin{array}{l}\text { The facilitator's role is the following: } \\
\text { - Ensure that everyone has a chance to speak and encourage everyone to expand on } \\
\text { certain points. Shy or reticent members may be encouraged by establishing eye } \\
\text { contact or by calling them by their names, and by asking them additional questions in } \\
\text { order to encourage useful information. } \\
\text { - Avoid dominating the discussion. } \\
\text { - Do not judge people who speak; accept what they say. } \\
\text { - Ask open questions, such as how, what, where, why as much as possible, especially to } \\
\text { clarify or to check understanding. Indeed, the role of the facilitator is only to boost } \\
\text { the conversation or confirm information; } \\
\text { - Listening and observing is a primordial quality to lead a focus group. The facilitator } \\
\text { should make a conscious effort to listen actively. He should seek more information by } \\
\text { observing non-verbal behaviours, taking in account the positioning of the participants } \\
\text { in the place where the FGD is conducted, how they organise themselves, who takes } \\
\text { the lead, who doesn't, who speaks, who doesn't, if some questions create discomfort } \\
\text { or conflicts, the way of speaking, ambiguous discourses, etc.; } \\
\text { Redirect the discussion in every case it is taken off topic; } \\
\text { - Manage the time. Estimate the time required for each topic and adapt it to the time } \\
\text { constraints of the group (whole FGD should take } 1.5 \text { - } 2 \text { hours); } \\
\text { Always try to avoid questions that presuppose certain answers. } \\
\text { The note-taker's role is the following: }\end{array}$ \\
\hline
\end{tabular}




\section{References}

Albadra, D., Vellei, M., Coley, D., \& Hart, J. (2017). Thermal comfort in desert refugee camps: An interdisciplinary approach. Building and Environment, 124, 460-477. https://doi.org/10.1016/j.build env.2017.08.016.

Albadra, D., Coley, D., \& Hart, J. (2018). RIBA president's awards for research 2017 winner of the annual theme - housing: Toward healthy housing for the displaced. Journal of Architecture, 23(1), 115-136. https://doi.org/10.1080/13602365.2018.1424227.

Alshawawreh, L., Sean Smith, R., \& Wood, J. (2017). Assessing the sheltering response in the middle east: Studying Syrian Camps in Jordan. International Journal of Social, Behavioral, Educational, Economic, Business and Industrial Engineering, 11(8), 2016-2022. https://doi.org/10.5281/zenodo.11315 51.

Alshawawreh, L., Pomponi, F., D’Amico, B., Snaddon, S., \& Guthrie, P. (2020). Qualifying the sustainability of novel designs and existing solutions for post-disaster and post-conflict sheltering. Sustainability, 12(3), 890. https://doi.org/10.3390/su12030890.

Atmaca, A., \& Atmaca, N. (2016). Comparative life cycle energy and cost analysis of post-disaster temporary housings. Applied Energy, 171, 429-443. https://doi.org/10.1016/j.apenergy.2016.03.058.

Beals, F., Kidman, J., \& Funaki, H. (2020). Insider and outsider research: Negotiating self at the edge of the Emic/Etic divide. Qualitative Inquiry, 26(6), 593-601.

Bekö, G., Lund, T., Nors, F., Toftum, J., \& Clausen, G. (2010). Ventilation rates in the bedrooms of 500 Danish children. Building and Environment, 45(10), 2289-2295. https://doi.org/10.1016/j.build env.2010.04.014.

Chowdhury, T., Chowdhury, H., Miskat, M. I., Chowdhury, P., Sait, S. M., Thirugnanasambandam, M., et al. (2020). Developing and evaluating a stand-alone hybrid energy system for Rohingya refugee community in Bangladesh. Energy, 191, 116568. https://doi.org/10.1016/j.energy.2019.116568.

D’Orazio, M., Stipa, P., Sabbatini, S., \& Maracchini, G. (2020). Experimental investigation on the durability of a novel lightweight prefabricated reinforced-EPS based construction system. Construction and Building Materials, 252, 119-134. https://doi.org/10.1016/j.conbuildmat.2020.119134.

Fosas, D., Albadra, D., Natarajan, S., \& Coley, D. (2018). Refugee housing through cyclic design. Architectural Science Review, 61(5), 327-337. https://doi.org/10.1080/00038628.2018.1502155.

Hart, J., Paszkiewicz, N., \& Albadra, D. (2018). Shelter as home? Syrian homemaking in Jordanian refugee camps. Human Organization, 77(4), 371.

IOM, International Organization for Migration. (2018). Shelter/ NFI Sector, Shelter Survey 2018. https:// www.humanitarianresponse.info/en/operations/bangladesh/document/shelter-survey-august-2018. Accessed 24 July 2020.

ISCG (2020). Bangladesh: Cox's bazar refugee population as of 31 May 2020. Inter Sector Coordination Group ISCG [online]. Available at: https://www.humanitarianresponse.info/sites/www.human itarianresponse.info/files/documents/files/20200616_cox_bazar_reference_map_with_pop_id022 5.pdf.Accessed 24 July 2020.

Kahn, J. S. (2014). Encountering extraordinary worlds: The rules of ethnographic engagement and the limits of anthropological knowing. Numen, 61(2/3), 237-54.

Klansek, T., Rota, F., Coley, D., Albadra, D., Paszkiewicz, N., Ball, R. (in press). Dataset for "Analysing experiences and issues in self-built shelters in Bangladesh using transdisciplinary approach. Bath: University of Bath Research Data Archive. https://doi.org/https://doi.org/10.15125/BATH-00781.

Kukadia, V., \& Upton, S. (2019). Ensuring good indoor air quality in buildings, BRE Trust, Bucknalls lane, Watford, Herts, WD25 9XX, March 2019.

Maynard, V., Parker, E., \& Twigg, J. (2016). The effectiveness and efficiency of interventions supporting shelter self-recovery following humanitarian crises: An evidence synthesis protocol. Oxford: Oxfam GB.

Pennise, D., Brant, S., Agbeve, S. M., Quaye, W., Mengasha, F., Tadele, W., et al. (2009). Indoor air quality impacts of an improved wood stove in Ghana and an ethanol stove in Ethiopia. Energy Sustain Dev, 14(2009), 71-76. https://doi.org/10.1016/j.esd.2009.04.003.

Pomponi, F., Moghayedi, A., Alshawawreh, L., D’Amico, B., \& Windapo, A. (2019). Sustainability of postdisaster and post-conflict sheltering in Africa: What matters? Sustainable Production and Consumption, 20(2019), 140-150. https://doi.org/10.1016/j.spc.2019.06.007.

Sphere. (2018). The Sphere Handbook: Humanitarian Charter and Minimum. Standards in Humanitarian Response, 4th ed., Geneva, Switzerland, 2018. www.spherestandards.org/handbook.

UNHCR. (2019). Briefing news: World's biggest refugee settlement gets biggest ever waste facility. https:// www.unhcr.org/news/briefing/2019/2/5c540fe74/worlds-biggest-refugee-settlement-gets-biggest-waste -facility.html. Accessed 24 July 2020. 
UNHCR. (2020a). Figures at glance. https://www.unhcr.org/uk/figures-at-a-glance.html. Accessed 17 Jan 202.

UNHCR. (2020b). Rohingya emergency. https://www.unhcr.org/uk/rohingya-emergency.html. Accessed 17 Jan 2020.

UNHCR \& REACH. (2018). Bangladesh: UNHCR camp settlement and protection profiling - Round 3 April 2018. https://data2.unhcr.org/en/documents/details/63821. Accessed 17 Jan 2020.

Vellei, M., Herrera, M., Fosas, D., \& Natarajan, S. (2017). The influence of relative humidity on adaptive thermal comfort. Building and Environment, 124, 171-185. https://doi.org/10.1016/j.build env.2017.08.005.

Wang, Y., Long, E., \& Deng, S. (2017). Applying passive cooling measures to a temporary disaster-relief prefabricated house to improve its indoor thermal environment in summer in the subtropics. Energy and Buildings, 139, 456-464. https://doi.org/10.1016/j.enbuild.2016.12.081.

WHO. (2010). Guidelines for Indoor Air Quality - Selected Pollutants (2010). The WHO European Centre for Environment and health, Bonn Office, WHO Regional Office for Europe coordinated the development of these WHO guidelines. ISBN 9789289002134.

WMO. (2020). World Meteorological Organisation - World weather information services - Chittagong, Bangladesh. https://worldweather.wmo.int/en/city.html?cityId=1164. Accessed 17 Jan 2020.

Publisher's Note Springer Nature remains neutral with regard to jurisdictional claims in published maps and institutional affiliations. 